\title{
Sesgo de respuesta y heterogeneidad en salud autopercibida. Evidencia del Panel de Hogares Británico
}

\author{
Cristina Hernández-Quevedo \\ European Observatory of Health Systems and Policies, London School \\ of Economics and Political Science, Reino Unido
}

Andrew M Jones

Department of Economics and Related Studies, University of York, Heslington

\author{
Nigel Rice
}

Centre for Health Economics, University of York, Reino Unido

\begin{abstract}
Resumen
Este artículo explora el sesgo de respuesta y heterogeneidad en la medida de salud autopercibida (SA) utilizada en el Panel de Hogares Británico (BHPS) y sus implicaciones para medir el gradiente socioeconómico en salud. La oleada 9 de la BHPS incluye el cuestionario general de salud SF-36, que incorpora un diferente formato de la pregunta de salud auto percibida utilizada en otras oleadas. En este estudio, se ha dedicado especial atención a la fiabilidad de SA y al grado de contaminación por error de medida; el cambio en la pregunta en la oleada 9 proporciona una forma de experimento natural que nos permite evaluar la sensibilidad de los análisis con datos de panel a un cambio en el instrumento de medida. En concreto, investigamos el sesgo de respuesta debido explícitamente a un cambio en la pregunta. Mostramos progresivamente cómo especificaciones generales de sesgo de respuesta pueden ser implementadas utilizando modelos probit ordenados y probit ordenados generalizados para datos de panel. Posteriormente, exploramos la sensibilidad de las medidas de desigualdad socioeconómica y de movilidad en salud a cambios en la medida de SA.
\end{abstract}

Palabras clave: salud autopercibida, sesgo de respuesta, probit ordenado, probit ordenado generalizado, desigualdad en salud

Clasificación JEL: I12 C23

\footnotetext{
Abstract

This paper explores reporting bias and heterogeneity in the measure of self-assessed health (SAH) used in the British Household Panel survey (BHPS) and their implications for measuring the socioeconomic gradiente in health. The ninth wave of the BHPS includes the SF-36 general health questionnaire,

Datos correspondientes al Panel de Hogares Británico (British Household Panel Survey, BHPS) fueron proporcionados por «ESRC Data Archive». Éstos no son responsables del análisis o interpretaciones presentados aquí. Este artículo procede del proyecto «La dinámica de la renta, salud y desigualdad en el ciclo de vida» (conocido como ECuity III Project), financiado en parte por el programa de Calidad de Vida y Gestión de Recursos de Vida de la Comunidad Europea (contrato QLK6-CT-2002-02297). Nigel Rice está financiado en parte por el programa de investigación del Departamento de Salud en el Reino Unido en el Centre for Health Economics, York. Los autores agradecen a Xander Koolman y Fabrice Etilé sus valiosas sugerencias, así como los comentarios aportados por los participantes en Health Economists'Study Group, Glasgow 2004, y HEDG Seminars, York.
} 
which incorporates a different wording to the self-assessed health variable used at other waves. Considerable attention has been devoted to the reliability of SAH and the scope for contamination by measurement error; the change in wording at wave 9 provides a form of natural experiment that allows us to assess the sensitivity of panel data analyses to a change in the measurement instrument. In particular, we investigate reporting bias due explicitly to the change in the question. We show how progressively more general specifications of reporting bias can be implemented using panel data ordered probit and generalised ordered probit models. Then we explore the sensitivity of measures of socioeconomic inequality and of mobility in health to changes in the measurement of $S A H$.

Key words: self-assessed health, reporting bias, ordered probit, generalised ordered probit, inequality in health.

JEL Classification: 112 C23

\section{Introducción}

La mayoría de las encuestas generales de población como el Panel de Hogares Británico (British Household Panel Survey, BHPS) incluyen una medida de salud autopercibida (SA): a los encuestados se les pide que evalúen su propia salud en una escala categórica de cinco puntos. En este estudio utilizamos once años del Panel de Hogares Británico, 1991-2001; esto proporciona la oportunidad de llevar a cabo un análisis longitudinal de la relación entre salud autopercibida y situación socioeconómica, permitiendo una investigación en profundidad de la dinámica o dependencia del estado de salud y la heterogeneidad individual. Sin embargo, los análisis longitudinales de salud autopercibida encaran un desafío - el cuestionario SF-36 fue incluido en la oleada 9 de la BHPS y, con ello, la pregunta de salud autopercibida fue reformulada, incluyendo además una modificación en las categorías de respuesta. Esto permite análisis multivariantes satisfactorios de la salud autopercibida que exploten la duración completa de los datos longitudinales. Con este desafío hay también una oportunidad. Se ha dedicado considerable atención a la fiabilidad de salud autopercibida y el alcance de contaminación por error de medida; el cambio en la pregunta en la oleada 9 proporciona una forma de experimento natural que nos permite evaluar la sensibilidad del análisis de datos de panel a un cambio en el instrumento de medida. Nos centramos en hasta qué punto el cambio en la pregunta de SA induce a sesgo de respuesta y qué forma toma cualquier sesgo de respuesta observado. En concreto, investigamos si diferentes grupos de individuos responden al cambio en el instrumento de medida de diferente forma. Si los encuestados respondieran de forma diferente, esto proporcionaría evidencia de diferencias sistemáticas en el comportamiento de respuesta. Esto tendría implicaciones para los análisis econométricos que utilizan SA como una variable dependiente o independiente y para el análisis de desigualdades en salud basado en SA. Por ejemplo, evidencia de sesgo de respuesta podría sugerir que los modelos probit ordenados de salud autopercibida son excesivamente restrictivos, imposibilitando una especificación más apropiada y flexible del efecto de los regresores en las probabilidades de resultados de salud. La estructura del artículo 
es la siguiente. La variable de salud autopercibida en el Panel de Hogares Británico se describe en la sección 2. La sección 3 describe el posible sesgo de respuesta y la estrategia de estimación y de prueba. La base de datos del Panel de Hogares Británico se describe en la sección 4. Los resultados se muestran en la Sección 5, concluyendo el artículo en la Sección 6.

\section{Salud autopercibida en el Panel de Hogares Británico}

La salud autopercibida se incluye con frecuencia en encuestas socioeconómicas generales, tales como el Panel de Hogares Británico y el Panel de Hogares de la Comunidad Europea (PHOGUE). La salud autopercibida ha sido utilizada ampliamente en estudios previos sobre la relación entre salud y situación socioeconómica (p.e., Adams et al. (2003), Benzeval et al. (2000), Deaton y Paxson (1998), Ettner (1996), Frijters et al. (2003), Salas (2002), Smith (1999)) y sobre la relación entre salud y formas de vida (p.e., Kenkel (1995), Contoyannis y Jones (2004)). La SA es una medida de salud subjetiva que proporciona un ranking ordinal del estado de salud percibido. Sin embargo, se ha mostrado que es un potente predictor de mortalidad subsiguiente (ver p.e., Idler y Kasl (1995), Idler y Benyamini (1997)) y su poder predictivo no parece variar entre grupos socioeconómicos (ver p.e., Burström y Fredlund (2001)). Medidas categóricas de SA se han revelado como buenos predictores de uso subsiguiente de atención médica (ver p.e., van Doorslaer et al. (2000), van Doorslaer, Koolman y Jones (2004)). Las desigualdades socioeconómicas en salud han sido también centro de investigación (ver p.e., van Doorslaer et al. (1997), van Doorslaer y Koolman (2004), van Ourti (2003)) y se ha mostrado que predicen las desigualdades en mortalidad (ver p.e., van Doorslaer y Gerdtham (2003)).

Varios estudios han analizado las implicaciones de incluir una referencia de edad en la pregunta de SA (Baron-Epel y Kaplan (2001), Kaplan y Baron-Epel (2003) y Eriksson et al (2004)). Baron-Epel y Kaplan (2001) analizan la «concordancia» entre dos medidas de SA: una pregunta general y una pregunta que incluye una referencia de edad. Los autores utilizan una encuesta de sección cruzada de adultos residentes en Israel (de 45-75 años de edad), basada en 793 entrevistas telefónicas. Los autores encuentran «poca concordancia» entre los individuos de mayor edad (65-75 años) sin enfermedades percibidas y aquellos individuos con menos de 12 años de educación y sin enfermedades percibidas. Estos dos grupos revelaron mejor salud cuando se les pidió comparar su salud con individuos de su propia edad y sexo. Sin embargo, una «concordancia excelente» se encontró entre las dos preguntas, para encuestados sin enfermedades de 55-65 años. Más años de educación se relacionó con evaluaciones más optimistas de salud, pero solo cuando se utilizó la medida de salud subjetiva relacionada con la edad. Los individuos proporcionaron diferentes evaluaciones de salud, particularmente los de mayor edad y los individuos con menor nivel educativo, incluso cuando compartían otras características tales como lengua, cultura, nacionalidad y religión. En un estudio posterior, Kaplan y Baron-Epel (2003) analizaron la inclusión de una re- 
ferencia de edad al preguntar a los individuos sobre su salud. De su muestra de 383 residentes israelíes, concluyeron que los individuos valoraban su salud comparando con personas de su edad, incluso cuando la referencia de edad no había sido incluida explícitamente en la pregunta de SA. Los autores consideran que los individuos entrevistados intentaron evaluar su salud de forma optimista. Por tanto, individuos mayores y con mala salud se compararon ellos mismos con individuos de su misma edad. Sin embargo, individuos jóvenes, pero con mala salud, evitaron compararse con aquéllos de su edad, para no sentirse en inferior condición. Eriksson et al (2004) comparan diferentes medidas de SA, teniendo en cuenta la inclusión de una referencia de edad en la pregunta. 8,200 individuos fueron proporcionados un cuestionario que incluía tres medidas de salud: dos medidas no comparables de SA, una con siete categorías de respuesta (SA-7) y una con cinco (SA-5), y una tercera medida que incluía una comparación con otros de la misma edad (SA-edad). Aunque los autores argumentan que las tres medidas proporcionan «evaluaciones paralelas de salud subjetiva», esto es, que pueden ser aplicadas de forma equivalente, los autores reconocen algunas diferencias entre las medidas. En particular, destaca el elevado nivel de falta de respuesta parcial para el grupo de mayor edad al responder SA-7, junto con la distribución menos sesgada para SA-edad, en comparación con las otras dos medidas.

Esta medida subjetiva de salud ha originado debate en la literatura respecto a su validez. Ha sido argumentado que la salud autopercibida no corresponde con la salud real (ver Bound, 1991), mientras otros investigadores han considerado esta variable como un indicador válido de salud verdadera (ver Butler et al., 1987). Como medida de salud autopercibida subjetiva, SA es propensa a errores de medida. Currie y Madrian (1999) y Lindeboom (2006) revisan evidencia general de error de medida no aleatoria en salud autopercibida. Crossley y Kennedy (2002) proporcionan evidencia de error de medida en una pregunta de SA con 5 categorías. Los autores explotan el hecho de que una sub-muestra aleatoria de los encuestados en la Encuesta Nacional de Salud Australiana de 1995 fueron preguntados la misma versión de la pregunta de SA dos veces, antes y después de otras preguntas sobre morbilidad. La primera pregunta fue administrada como parte del cuestionario SF-36 en un formulario auto-administrado, la segunda como parte de una entrevista cara a cara en el cuestionario principal. Se encontró una diferencia estadísticamente significativa en la distribución de SA entre las dos preguntas y evidencia que estas diferencias están relacionadas con edad, renta y ocupación. Este error de medida podría ser explicado por un efecto de modo de administración, debido al uso de entrevistas de auto-administración y cara a cara (Grootendorst et al. (1997) encuentran que las preguntas de auto-administración revelan más morbilidad); o un efecto contextual o de aprendizaje por el cual las respuestas de SA están influenciadas por las cuestiones de morbilidad incluidas.

Con frecuencia se argumenta que la asociación entre «salud verdadera» y las categorías de SA podría variar con las características de los encuestados. Esta fuente de error de medida se le conoce como sesgo de respuesta dependiente del estado (statedependent reporting bias) (Kerkhofs y Lindeboom (1995)), escala de sesgo de referencia (scale of reference bias) (Groot (2000)) y como como un desplazamiento de 
los puntos de corte de la categoría de respuesta (response category cut-point shift) (Sadana et al. (2000), Murray et al. (2001)). Esto ocurre si los subgrupos de la población utilizan sistemáticamente diferentes niveles de puntos de corte al revelar su SA, a pesar de tener el mismo nivel de «salud verdadera».

El análisis de regresión de SA puede ser logrado a través de la especificación de un modelo de probabilidad ordenado, tal como el probit o logit ordenado. En el contexto de los modelos probit ordenados, los síntomas de error de medida pueden ser capturados haciendo que los puntos de corte dependan de algunas o de todas las variables exógenas utilizadas en el modelo y estimando un probit ordenado generalizado. Esto requiere fuertes restricciones a priori sobre cuáles son las variables que afectan a la salud y cuáles afectan las respuestas, para indentificar separadamente la influencia de variables en la salud latente y en el error de medida. Es valioso destacar que permitir que la escala de SA varíe entre características individuales es equivalente a una especificación heterocedástica de la ecuación de la variable latente subyacente (ver p.e., van Doorslaer y Jones (2003)). Esto se debe a que la localización y la escala no pueden ser identificadas separadamente en modelos de elección binaria y ordenados y, en general, no es posible separar el error de medida de la heterogeneidad. Intentos para superar este problema fundamental de identificación incluyen la modelización del sesgo de respuesta basado en indicadores más «objetivos» de salud verdadera (Kerkhofs y Lindeboom (1995), Lindeboom y van Doorslaer (2004)) y el uso de «viñetas» para fijar la escala (Kapteyn et al 2004; Murray et al., 2001)). Lindeboom y van Doorslaer (2004) analizan SA en la Encuesta Nacional de Salud de la Población correspondiente a Canadá y utilizan el Índice de Utilidad de Salud McMaster (HUI-3) como su medida objetiva de salud. Encuentran evidencia de desplazamiento de los puntos de corte con respecto a edad y género, pero no para la renta, educación o grupos lingüísticos.

En general, la pregunta de SA se mide siguiendo una escala ordinal, con posibles respuestas que van desde «muy mala»o «mala» hasta «muy buena»o «excelente» salud. La variable de SA que está incluida en el Panel de Hogares Británico (BHPS) ha sido planteada de dos formas diferentes, dependiendo de la oleada. Para las oleadas 1-8 y 10 en adelante, la variable SA representa "estado de salud durante los últimos 12 meses». Los individuos son preguntados: «Comparado con los individuos de su misma edad, diría que su salud durante los últimos 12 meses en su totalidad ha sido: excelente, buena, regular, mala, muy mala?». Sin embargo, el cuestionario SF-36 fue incluido en la oleada 9. El cuestionario SF-36 incluye 36 elementos que miden la salud a través de ocho dimensiones de funcionamiento físico, funcionamiento social, limitaciones de movimiento debido a problemas físicos, limitaciones de movimiento debido a problemas emocionales, salud mental, vitalidad, dolor y percepciones generales de salud (Jenkinson et al., 1996). En este cuestionario, la variable SA para la oleada 9 representa «estado general de salud», utilizando la pregunta: «En general, diría que su salud es: excelente, muy buena, buena, regular, mala?».

Si las dos preguntas son comparadas, es posible distinguir tres diferencias principales. En primer lugar, la pregunta de salud autopercibida en la oleada 9 no inclu- 
ye la referencia de edad que está presente en el resto de las oleadas, en las cuales a los individuos se les pide evaluar su nivel de salud «comparado con los individuos de su misma edad». El segundo es el marco de referencia: a los encuestados en las oleadas diferentes a la 9 se les proporciona un período de referencia de 12 meses, mientras que a aquéllos en la oleada 9 no se les proporciona dicha referencia. La tercera diferencia es la forma en la cual las categorías han sido identificadas. Aunque ambas preguntas proporcionan cinco posibles respuestas a los encuestados, la categoría «muy mala» no está disponible en la oleada 9 , pero «muy buena» es incorporada entre «buena» $\mathrm{y}$ «excelente».

La Figura 1 presenta la distribución de SA para cada oleada, utilizando un panel equilibrado de individuos que son observados durante todas las 11 oleadas. Por consiguiente, todas las distribuciones específicas de cada oleada están basadas en la misma muestra de individuos seguidos en el tiempo. Las diferentes categorías se muestran en el eje horizontal de cada gráfico, con «1» representando el nivel más bajo de salud $^{1}$, y «5», el nivel más elevado ${ }^{2}$. Los histogramas para las oleadas 1-8 y 10-11

\section{FIGURA 1}

\section{DISTRIBUCIÓN DE SA PARA CADA OLEADA}
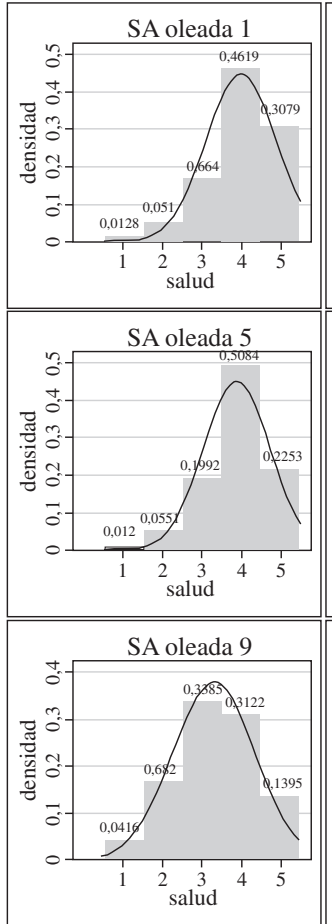
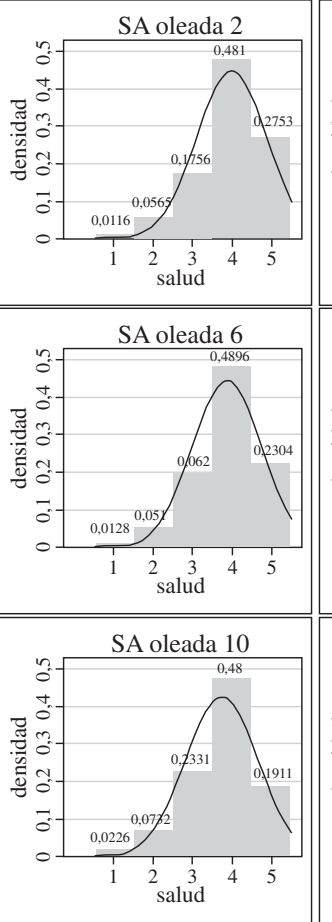
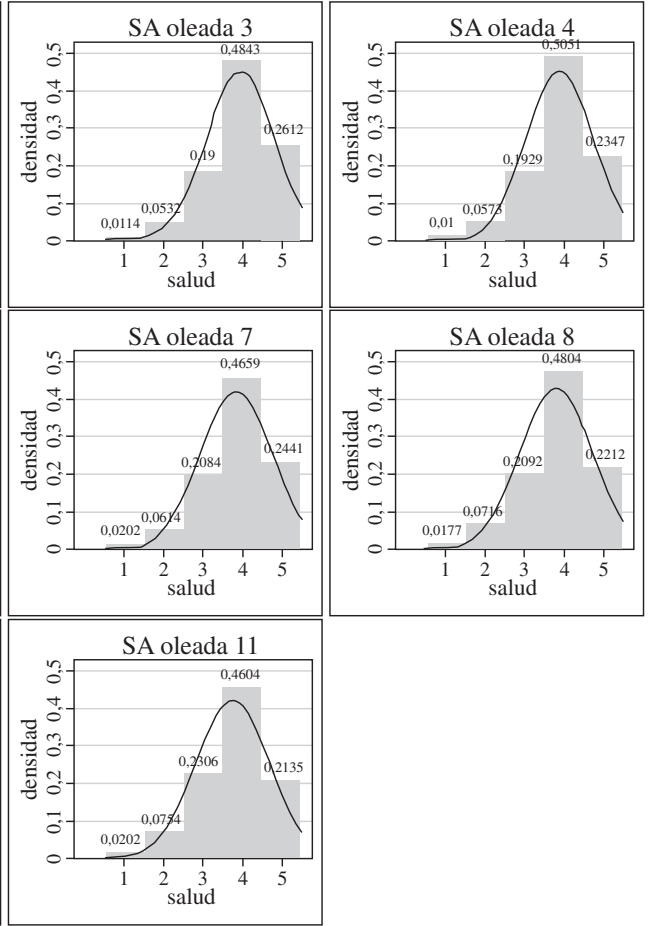

Esto corresponde a «muy mala» salud en las oleadas 1-8, 10-11 y «mala» salud en oleada 9.

2 Corresponde a «excelente» salud en todas las oleadas. 
siguen un patrón similar; una distribución sesgada es clara, con la mayoría de los encuestados considerando su salud como «buena» o «excelente». Alrededor del 50 por ciento de individuos revelan una «buena» salud y alrededor del 70 por ciento, o bien «buena» o «excelente» salud. Estos porcentajes varían muy poco entre oleadas. Destacar que aunque la pregunta está enmarcada en términos de auto-valoración de salud comparado con individuos de su misma edad, la distribución de las respuestas entre las 10 oleadas indica un empeoramiento de la salud percibida mientras que el cohorte envejece: por ejemplo, $5 \%$ de los individuos revelan «mala» salud en la oleada 1 comparado con el 7.5\% en la oleada 11 y $30 \%$ de los encuestados perciben «excelente» salud en la oleada 1 comparado con el $21 \%$ en la oleada 11.

En la oleada 9, donde la versión de SA procedente del cuestionario SF-36 fue utilizada, hay un notable cambio en la distribución de las respuestas. Menos individuos califican su estado de salud dentro de las dos categorías más altas («excelente» y «muy buena» salud), mientras que una mayor proporción de encuestados la califican dentro de las dos categorías inferiores (salud «mala» y «regular»). En general, parece existir un desplazamiento hacia la izquierda de la distribución junto con una compresión de sus puntos de masa. Hay también evidencia de un «efecto suelo»: los individuos que revelan «mala» salud en la oleada 9, podrían haber evaluado su salud como «muy mala» si esta categoría hubiera estado disponible.

\section{Modelos multivariantes para respuestas ordenadas en datos longitudinales}

\subsection{Sesgo de respuesta}

Dado el cambio en la distribución de la variable de SA en la oleada 9, sesgo de respuesta podría ser considerado como una explicación para esta observación. El sesgo de respuesta ha sido una preocupación en la literatura y podría ser definido como el diferencial de percepción de salud entre individuos o grupos de individuos con el mismo estado salud. El uso sistemático de diferentes umbrales por parte de subgrupos de una población reflejan la existencia de sesgo de respuesta ((Lindeboom y van Doorslaer, 2004; Murray et al, 2001; Groot, 2000; Sadana et al, 2000; Kerkhofs y Lindeboom, 1995). Estas diferencias podrían están influenciadas por, entre otras cosas, edad, género, educación, renta, lengua y experiencia personal de enfermedad. Básicamente, significa que diferentes grupos parecen interpretar la pregunta dentro de su propio contexto específico y por tanto utilizan deferentes puntos de referencia cuando están respondiendo a la misma pregunta.

En el análisis de sesgo de respuesta realizado por Lindeboom y van Doorslaer (2004), los autores distinguen entre desplazamiento del índice (index shift) y desplazamiento de los puntos de corte (cut-point shift). El primero ocurre si la forma de la distribución de SA se mantiene, pero hay un cambio en su localización de tal forma que hay un desplazamiento paralelo en todos los umbrales de respuesta para subgrupos concretos de la población. Esto se ilustra en la Figura 2: 
FIGURA 2
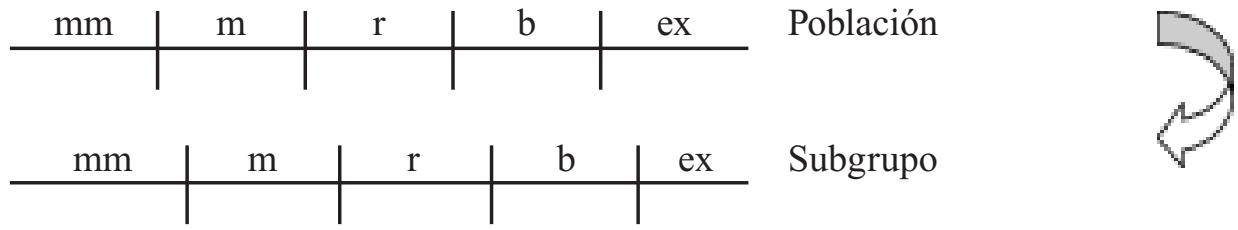

Se debería destacar que el término «desplazamiento del índice» podría ser engañoso. El desplazamiento paralelo en la distribución se podría deber a un desplazamiento en los puntos de corte (sesgo de respuesta) o debido a un desplazamiento en la medida latente de «salud verdadera». En general, no es posible identificar separadamente las dos razones de «desplazamiento del índice».

Si el sesgo de respuesta se debe al desplazamiento de los puntos de corte, el segundo caso, esto implica que hay un cambio en las posiciones relativas de los umbrales de respuesta para subgrupos particulares de la población (ver Figura 3 como ejemplo). Esto resultaría en un cambio en la distribución total de SA.

FIGURA 3

\begin{tabular}{l|c|c|c|cc}
$\mathrm{mm}$ & $\mathrm{m}$ & $\mathrm{r}$ & $\mathrm{b}$ & $\mathrm{ex}$ & Población \\
& & & & & \\
$\mathrm{mm}$ & $\mathrm{m}$ & $\mathrm{r}$ & $\mathrm{b}$ & $\mathrm{ex}$ & Subgrupo
\end{tabular}

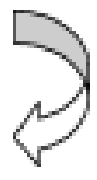

Una comparación de las dos versiones de la pregunta de SA en el Panel de Hogares Británico tiene la complejidad añadida de la versión de SA correspondiente al cuestionario SF-36 en la oleada 9, presentando una pregunta planteada de forma diferente al igual que un conjunto de categorías de respuesta diferentes, comparado con la pregunta realizada en las oleadas 1-8 y 10-11. Las diferencias resultantes en la distribución de SA en la oleada 9 comparado con la distribución en las otras oleadas podrían ser debido a, o bien un «desplazamiento del índice» en el ajuste de salud verdadera con las categorías de SA o a un cambio en la localización relativa de los puntos de corte. La Figura 4 ilustra un desplazamiento en los puntos de corte en la oleada 9.

\section{FIGURA 4}
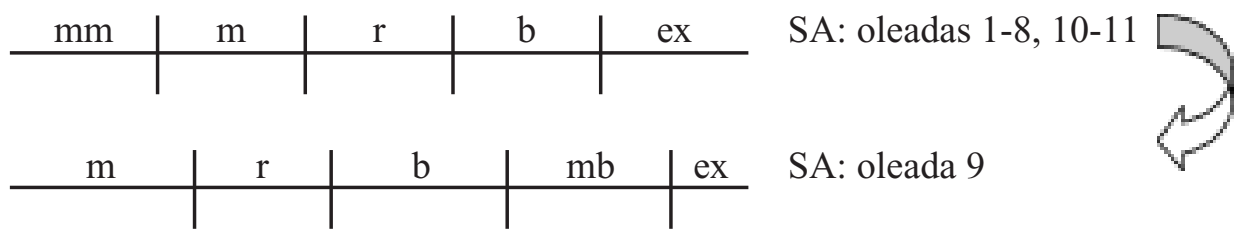
La Figura 1 muestra evidencia de que, al menos a nivel agregado, los individuos parecen retener una interpretación razonablemente consistente de la pregunta original de SA. Esto es, las distribuciones de las respuestas a la pregunta de SA en las oleadas 1-8 y 10-11 son muy similares. Sin embargo, hay una tendencia a revelar una salud que empeora durante las 11 oleadas. Esto podría ser indicativo de un deterioro de la salud verdadera mientras que la cohorte envejece o bien podría ser debido a un desplazamiento de los umbrales de respuesta utilizados por los encuestados. La distribución de SA en la oleada 9 claramente difiere de las distribuciones observadas en otras oleadas. El efecto conjunto de un cambio en el marco de la pregunta junto con la adición de la categoría «muy buena» y la eliminación de la categoría «muy mala», es la fuente más probable de la mayoría de las diferencias observadas $^{3}$. Es de interés comprobar si estos efectos han resultado en o bien en un desplazamiento del índice o en un desplazamiento en los puntos de corte en la relación que ajusta salud verdadera latente con las categorías observadas de SA.

\subsection{Probit ordenado}

Uno de los objetivos de este artículo es comprobar si el cambio en la medida de SA en la oleada 9 del Panel de Hogares Británico influencia los estimadores de modelos de datos de panel para respuestas ordenadas y qué nos puede decir esto sobre error de medida. Para este propósito, modelos probit ordenados (pooled probit) han sido aplicados. La literatura sugiere que los modelos probit o logit ordenados pueden ser utilizados cuando consideramos una variable categórica ordenada, tales como SA (McElvey y Zavoina, 1975), y si la escala de la variable está disponible, la regresión por intervalos es una alternativa (van Doorslaer y Jones, 2003). El modelo probit es estimado con errores estándares robustos, permitiendo la agrupación de las observaciones entre individuos (ver p.e., Wooldridge, 2002).

Los modelos probit (y logit) ordenados suelen estar motivados por la especificación de una variable latente: esto hace una distinción explícita entre el proceso que determina la salud verdadera del individuo —el modelo de variable latente - y los umbrales que determinan qué categoría de SA revelan, dada su verdadera salud —el modelo de medida. La especificación de la variable latente para los modelos considerados en este estudio viene dada por:

$$
h_{i t}^{*}=\beta^{\prime} x_{i t}+\varepsilon_{i t} \quad i=1, \ldots, N ; t=1, \ldots, T
$$

donde $\varepsilon_{i t}$ es un término de error idiosincrático no correlacionado con el conjunto de regresores $x_{i t}, h_{i t}{ }^{*}$ representa el nivel de salud verdadero para el individuo i en el mo-

3 Es posible que la distribución de «verdadera» salud en la oleada 9 difiera de la distribución de «verdadera» salud en las otras oleadas y que esto aparezca reflejado en el estado de salud autopercibida irrespectivamente del marco de la pregunta. Sin embargo, de acuerdo con la evidencia sobre la consistencia en la distribución percibida de SA con las otras oleadas, esto parecería altamente improbable. 
mento t. Como salud verdadera no es observable, nos basamos en el uso de SA como una variable dependiente apropiada. Es útil destacar que la consistencia del estimador probit se basa en la premisa que $x_{i t}$ es independiente del error latente, $\varepsilon_{i t}$, en cada período de tiempo. El modelo probit ordenado proporciona coeficientes promedios a nivel poblacional (Zeger et al., 1988). El modelo probit ordenado asume que el término de error, $\varepsilon_{i t}$, se distribuye como $\mathrm{N}(0,1)$ para la identificación de $\beta$ (ver p.e., Wooldridge, 2002, p.486).

El modelo de medida relaciona valores de $h_{i t}{ }^{*}$ con las categorías percibidas de SA. Si tenemos en cuenta los dos formatos de la variable de SA en la oleada 9, la especificación de la variable latente sería la siguiente ${ }^{4}$ :

- Para el formato original de la pregunta, i.e., para las oleadas 1-8 y 10-11, $h_{i t}=j$ si $\mu_{j-1}^{0}<h^{*}{ }_{i t}<\mu_{j}^{0}$, con $\mathrm{j}=1$ (muy mala), 2 (mala), 3 (regular), 4 (buena), 5 (excelente)

- En el caso del formato de la pregunta en la oleada 9, $h_{i t}=j$ si $\mu_{j-1}^{l}<h_{i t}^{*}<\mu_{j}^{l}$, con j = 1 (mala), 2 (regular), 3 (buena), 4 (muy buena), 5 (excelente)

Dado este cambio en el fraseado de la pregunta, la probabilidad de que el individuo i elija la categoría j vendrá dada por la siguiente expresión:

$$
\begin{array}{rlrl}
P\left(h_{i j}=j \mid x_{i j}\right)= & & \\
& \Phi\left(\mu_{j}^{0}-\beta^{\prime} x_{i t}\right)-\Phi\left(\mu_{j-1}^{0}-\beta^{\prime} x_{i t}\right) & & t=1-8,10-11 \\
& \Phi\left(\mu_{j}^{1}-\beta^{\prime} x_{i t}\right)-\Phi\left(\mu_{j-1}^{1}-\beta^{\prime} x_{i t}\right) & & t=9
\end{array}
$$

Se observa que los coeficientes $\beta$ 's son comunes en todas las oleadas; dada la especificación de la variable latente, estos parámetros no deberían estar afectados por cambios en el modelo de medida. Esta hipótesis proporciona la base de nuestros tests de sesgo de respuesta. El interés se centra en los estimadores de los puntos de corte $\mu_{j}^{0}$ y $\mu_{j}^{l}$.

\subsection{Tests Empíricos}

La forma más simple de «desplazamiento del índice» aparece donde hay un desplazamiento paralelo en los umbrales de respuesta, común a todos los individuos. Esto implica que los puntos de corte originales $\left(\mu_{j}^{0}\right)$ pueden ser obtenidos añadiendo una constante, $\gamma$, a los puntos de corte correspondientes a la oleada $9\left(\mu_{j}^{l}\right)$ :

$$
\mu_{j}^{0}=\mu_{j}^{t}+\gamma, \quad \text { para todos } j
$$

4 Para identificar el modelo, seguimos STATA al utilizar la normalización convencional, ésta es: $\mu_{0}^{0}=-\infty, \mu_{5}^{0}=+\infty$ y no hay término de constante en el índice lineal dado por la ecuación (1). 
En este caso particular, la probabilidad que un individuo i elija la categoría j para evaluar su salud en el momento t, puede ser escrita como:

$$
\begin{array}{rlrl}
P\left(h_{i t}=j \mid x_{i t}\right)= & & \\
& \Phi\left(\mu_{j}^{0}-\beta^{\prime} x_{i t}\right)-\Phi\left(\mu_{j-1}^{0}-\beta^{\prime} x_{i t}\right) & & t=1-8,10-11 \\
& \Phi\left(\mu_{j}^{0}-\gamma-\beta^{\prime} x_{i t}\right)-\Phi\left(\mu_{j-1}^{1}-\gamma-\beta^{\prime} x_{i t}\right) & & t=9
\end{array}
$$

Esto muestra que el desplazamiento paralelo en la oleada 9 puede ser capturado añadiendo un indicador para la oleada 9 en la ecuación de la variable latente el desplazamiento paralelo en los puntos de corte en (3) es equivalente a un desplazamiento en la intercepción de una ecuación de la variable latente (1) en la oleada 9, y por tanto la identificación «desplazamiento del índice» utilizada por Lindeboom y van Doorslaer (2004). Sin embargo, en el contexto de nuestra aplicación al Panel de Hogares Británico se mantiene la premisa que la ecuación de verdadera salud, (1), no cambia en la oleada 9 sino el modelo de medida, (2). Así que una indicación más apropiada sería «desplazamiento paralelo de puntos de corte». En la práctica, los indicadores para cada año son incluidos para cada oleada del panel y el interés está en si la variable para la oleada 9 destaca o se distingue de la tendencia subyacente.

Este argumento puede ser extendido para el caso donde el «desplazamiento del índice» difiere entre diferentes tipos de individuos, definidos por sus características $x$ medidas en la oleada 9:

$$
\mu_{j}^{0}=\mu_{j}^{t}+\delta^{\prime} x_{i 9}, \quad \quad \text { para todos } j
$$

Siguiendo el mismo argumento presentado anteriormente, el modelo puede ser reformulado de tal forma que la variable latente se expresa como sigue:

$$
\begin{array}{rlrl}
h_{i t}^{*} & =\beta^{\prime} x_{i t}+\varepsilon_{i t} & & t=1-8,10-11 \\
h_{i t}^{*} & =(\beta+\delta)^{\prime} x_{i t}+\varepsilon_{i t} & t & t=9
\end{array}
$$

En este caso, las diferencias en respuesta en la oleada 9 son capturadas por la inclusión de términos de interacción entre el indicador de la oleada 9 y las variables explicativas. Mientras que el desplazamiento en los umbrales de respuesta en la oleada 9 es una función de características individuales, el mismo desplazamiento se aplica a cada uno de los umbrales.

Nuestra especificación se basa en la distinción entre el modelo de variable latente (1) y el modelo de medida. En particular, hemos asumido que hay un índice único que describe salud «verdadera». Esto podría ser relajado permitiendo una especificación de probit ordenado generalizado, en el cual a las $\beta$ 's se les permite va- 
riar entre las categorías percibidas de $\mathrm{SA}\left(\beta_{\mathrm{j}}, \mathrm{j}=1 \ldots 5\right)$. Esta especificación generalizada tiene sentido intuitivo si las categorías ordenadas reflejan elecciones disponibles para el individuo, pero este no es el caso con salud autopercibida. Las categorías de SA son un artefacto del diseño de la pregunta en la encuesta y es complicado construir un argumento de que la relación entre la salud verdadera de alguien y sus características socioeconómicas sean independientes al número e identificación de estas categorías arbitrarias. Sin embargo, la existencia de heterogeneidad individual en las $\beta$ 's (i.e., permitiendo que $\beta_{\mathrm{i}}$ difiera entre individuos) tal que, por ejemplo, la asociación entre renta y salud es más baja para aquellos con mejor salud, podría llevar a heterogeneidad entre categorías de SA (ver van Doorslaer y Jones, 2003). Para evaluar la hipótesis de índice único - algunas veces referida como la hipótesis de proporcionalidad (ver Long, 1997, p.140), estimamos las especificaciones para probit ordenados con diferentes números de categorías de SA. Además de 5 categorías, también estimamos modelos con SA reducida a 4 y 3 categorías. El interés se centra en si las $\beta$ 's se mantienen estables con diferentes categorizaciones de la variable dependiente. Si es el caso, entonces esto proporciona evidencia que nuestro modelo de variable latente subyacente es consistente con los datos.

Este enfoque también revela información sobre el «desplazamiento de los puntos de corte». Esto ocurre cuando el desplazamiento en los umbrales específicos puede diferir. En general no es posible distinguir entre heterogeneidad y error de medida (ver p.e., van Doorslaer y Jones, 2003). Sin embargo, una comparación de especificaciones con 5, 4 o 3 categorías proporcionará evidencia sobre si los coeficientes se mantienen estables entre categorías. Si son estables, podemos razonablemente asumir que cualquier desplazamiento del punto de corte observado se debe a error de medida en vez de heterogeneidad en la salud latente.

El análisis previo puede ser extendido, para comprobar el desplazamiento en los puntos de corte. Para ese propósito, nosotros estimamos un modelo permitiendo que los parámetros puedan variar entre las categorías de $y$ para cada oleada, siguiendo un modelo probit ordenado generalizado (POG). El POG permite la relajación de una de las características restrictivas del modelo probit ordenado: la invariabilidad de los umbrales (Terza, 1985). Para permitir que los puntos de corte varíen con los regresores, $\mu_{j}^{0}$ necesita ser reformulado como sigue:

$$
\mu_{i j}^{0}=\mu_{j}^{0}+\mu_{j}^{0} x_{i t} \quad \text { para todos } j
$$

Si el impacto de las características socioeconómicas en los umbrales en la oleada 9 difiere entre umbrales, (5) se convierte en:

$$
\mu_{i r}^{0}=\mu_{i j}^{1}+\delta_{j}^{1}, x_{i 9}, \quad \text { para } t=9
$$

Entonces, $\delta_{j}^{l}$ en la ecuación (8) representa el efecto adicional de los regresores en los puntos de corte. Dados (1), (7) y (8): 


$$
\begin{array}{rlrl}
P\left(h_{i t}=j \mid x_{i j}\right)= & \\
& \Phi\left(\mu_{j}^{0}-\left(\beta-\delta_{j}^{0}\right)^{\prime} x_{i t}\right)-\Phi\left(\mu_{j-1}^{0}-\left(\beta-\delta_{j}^{0}\right)^{\prime} x_{i t}\right) & & t=1-8,10-11 \\
& \Phi\left(\mu_{j}^{0}-\left(\beta-\delta_{j}^{t}-\delta_{j}^{0}\right)^{\prime} x_{i t}\right)-\Phi\left(\mu_{j-1}^{0}-\left(\beta+\delta_{j}^{t}-\delta_{j}^{0}\right)^{\prime} x_{i t}\right) & & t=9
\end{array}
$$

Esto resulta en una especificación en la que los coeficientes varían entre categorías de SA, como resultado del desplazamiento de los puntos de corte. El POG es estimado directamente por máxima verosimilitud, con errores estándares robustos corregidos agrupando entre individuos. Para la identificación, se impone la normalización $\mu_{i 4}^{0}=\mu_{4}^{0}$, tal que el desplazamiento de los puntos de corte asociados con las $x$ 's es medido con relación a $\mu_{4}^{0}$.

\section{El Panel de Hogares Británico}

El Panel de Hogares Británico es una encuesta longitudinal de hogares privados en Gran Bretaña (Inglaterra, Gales y Escocia al sur del Canal de Caledonia), y ha sido diseñada como una encuesta anual de cada adulto $(16+)$ miembro de una muestra nacionalmente representativa de más de 5,000 hogares, con un total aproximadamente de 10,000 entrevistas individuales. La primera oleada de la encuesta fue conducida entre el 1 de Septiembre de 1990 y el 30 de Abril de 1991. La selección inicial de hogares para la inclusión en la encuesta fue realizada utilizando un procedimiento de muestreo sistemático estratificado a dos niveles, diseñado para dar a cada dirección un probabilidad de selección aproximadamente igual ${ }^{5}$. Los mismos individuos son re-entrevistados en oleadas sucesivas y, si se separan de los hogares originales, son también re-entrevistados conjuntamente con todos los miembros adultos de sus nuevos hogares.

Un panel equilibrado es utilizado, de tal forma que solo los individuos de la primera oleada que fueron entrevistados en cada oleada consecutiva son incluidos. Siguiendo a Contoyannis et al. (2004), incluimos individuos que proporcionaron una entrevista completa en cada oleada ${ }^{6}$. Nuestra muestra de trabajo consiste en 2,255 hombres y 2,841 mujeres. Todos los modelos son estimados separadamente para hombres y mujeres.

\section{Salud autopercibida}

Las dos versiones de SA disponibles en el Panel de Hogares Británico han sido descritas en la sección 2. Para ambas preguntas, las respuestas son codificadas en orden creciente de salud. Por ejemplo, para las oleadas 1-8 y 10-11, «muy mala» sa-

5 Para más detalles ver Taylor (1998).

${ }^{6}$ Esto excluye a individuos para los cuales se proporcionaron entrevistas proxy junto con aquéllos que respondieron a entrevistas telefónicas. 
lud está codificada como «1», mientras que «excelente» salud está codificada como «5». En la oleada 9, «mala» salud está codificada como «1» y «excelente» salud como «5».

Para evaluar la hipótesis de índice único, creamos una nueva variable de SA de tal forma que haya apoyo común dentro de cada categoría entre las dos versiones de la pregunta. Por ejemplo, primero reducimos la variable SA en cuatro categorías creando una nueva variable de SA con los códigos siguientes: 1: los individuos que percibieron «muy mala» $\mathrm{o}$ «mala» salud en las oleadas 1-8 y 10-11, o individuos que percibieron «mala» salud en la oleada 9; 2 : individuos que perciben salud «regular»; 3: individuos que perciben «buena» salud en las oleadas 1-8 y 10-11, o «buena»o «muy buena» salud en la oleada 9; 4: individuos que revelan «excelente» salud. Las categorías de la SA original fueron también reducidas a una variable de tres categorías. Las categorizaciones reducidas están resumidas en la Tabla 1.

Se presenta también una construcción adicional de SA que contiene 4 categorías. Esto permite aplicar un enfoque sugerido por Lindley y Lorgelly (2003). Estos autores estiman la relación entre la desigualdad en renta y salud autopercibida utilizando las primeras nueve oleadas del Panel de Hogares Británico. El interés de su artículo para el propósito de este estudio es la forma en la que los datos de la oleada 9 son incluidos en el análisis. En concreto, SA ha sido construida como una variable categórica con 4 categorías, recodificadas como «excelente», «buena», «regular»y «mala». «Mala» y «muy mala» fueron unidas en la nueva categoría de «mala». En la oleada 9 los encuestados que perciben «muy buena» salud fueron reasignados a «buena» 0 «excelente» salud de la siguiente forma. Una muestra aleatoria conformada por el 33\% de individuos fue recodificada como «excelente». El resto de individuos fue

\section{TABLA 1}

CONSTRUCCIÓN DE LA VARIABLE SA

\begin{tabular}{|c|c|c|c|c|}
\hline Oleada & Categoría & $\begin{array}{c}\text { SA 5 } \\
\text { categorías }\end{array}$ & $\begin{array}{c}\text { SA } 4 \\
\text { categorías }\end{array}$ & $\begin{array}{c}\text { SA 3 } \\
\text { categorías }\end{array}$ \\
\hline $1-8,10-11$ & $\begin{array}{l}1 \\
2 \\
3 \\
4 \\
5\end{array}$ & $\begin{array}{l}\text { muy mala } \\
\text { mala } \\
\text { regular } \\
\text { buena } \\
\text { excelente }\end{array}$ & $\begin{array}{l}\text { muy mala, mala } \\
\text { regular } \\
\text { buena } \\
\text { excelente }\end{array}$ & $\begin{array}{c}\text { muy mala, mala } \\
\text { regular } \\
\text { buena, excelente }\end{array}$ \\
\hline 9 & $\begin{array}{l}1 \\
2 \\
3 \\
\\
4 \\
5\end{array}$ & $\begin{array}{l}\text { mala } \\
\text { regular } \\
\text { buena } \\
\text { muy buena } \\
\text { excelente }\end{array}$ & $\begin{array}{l}\text { mala } \\
\text { regular } \\
\text { buena, } \\
\text { muy buena } \\
\text { excelente }\end{array}$ & $\begin{array}{c}\text { mala } \\
\text { regular } \\
\text { buena, muy buena } \\
\text { excelente }\end{array}$ \\
\hline
\end{tabular}


recodificado como «buena». Una muestra aleatoria del $20 \%$ de individuos percibiendo «buena» salud en la oleada 9 fue recodificado como «regular». Además, una muestra aleatoria de individuos que revelan salud «regular» en la oleada 9 fue recodificada como «mala». Los autores argumentan que estas recodificaciones y pesos fueron elegidos con la intención de mantener promedios de cada categoría de salud autopercibida, para todas las oleadas (Lindley y Lorgelly, 2003). La Tabla 2, presenta la proporción de encuestados en cada categoría para las varias construcciones de la variable de SA. Una comparación de las frecuencias observadas entre las once oleadas para las cuatro clasificaciones de la variable SA, muestra que el enfoque adoptado por Lindley y Lorgelly (2003) proporciona una mayor consistencia. En particular, las frecuencias para cada una de las categorías observadas en la oleada 9 están cerca de aquéllas obtenidas por las mismas categorías en otras oleadas.

TABLA 2

FRECUENCIAS PARA LAS CLASIFICACIONES DE SA

\begin{tabular}{|l|c|c|c|c|c|c|c|c|c|c|c|}
\hline \multicolumn{10}{|c|}{ Oleada } \\
\hline & $\mathbf{1}$ & $\mathbf{2}$ & $\mathbf{3}$ & $\mathbf{4}$ & $\mathbf{5}$ & $\mathbf{6}$ & $\mathbf{7}$ & $\mathbf{8}$ & $\mathbf{9}$ & $\mathbf{1 0}$ & $\mathbf{1 1}$ \\
\hline SA 5 & & & & & & & & & & & \\
1 & 1,28 & 1,16 & 1,14 & 1,00 & 1,20 & 1,28 & 2,02 & 1,77 & 4,16 & 2,26 & 2,02 \\
2 & 5,10 & 5,65 & 5,32 & 5,73 & 5,51 & 6,20 & 6,14 & 7,16 & 16,8 & 7,32 & 7,54 \\
3 & 16,6 & 17,6 & 19,0 & 19,3 & 19,9 & 20,5 & 20,8 & 20,9 & 33,9 & 23,3 & 23,1 \\
4 & 46,2 & 48,1 & 48,4 & 50,5 & 50,8 & 49,0 & 46,6 & 48,0 & 31,2 & 48,0 & 46,0 \\
5 & 30,8 & 27,5 & 26,1 & 23,4 & 22,5 & 23,0 & 24,4 & 22,1 & 14,0 & 19,1 & 21,4 \\
\hline SA 4 & & & & & & & & & & & \\
1 & 6,38 & 6,81 & 6,46 & 6,73 & 6,71 & 7,48 & 8,16 & 8,93 & 4,16 & 9,58 & 9,56 \\
2 & 16,6 & 17,6 & 19,0 & 19,3 & 19,9 & 20,5 & 20,8 & 20,9 & 16,8 & 23,3 & 23,1 \\
3 & 46,2 & 48,1 & 48,4 & 50,5 & 50,8 & 49,0 & 46,6 & 48,0 & 65,1 & 48,0 & 46,0 \\
4 & 30,8 & 27,5 & 26,1 & 23,5 & 22,5 & 23,0 & 24,4 & 22,1 & 14,0 & 19,1 & 21,4 \\
\hline SA 3 & & & & & & & & & & & \\
1 & 6,38 & 6,81 & 6,46 & 6,73 & 6,71 & 7,48 & 8,16 & 8,93 & 4,16 & 9,58 & 9,56 \\
2 & 16,6 & 17,6 & 19,0 & 19,3 & 19,9 & 20,5 & 20,8 & 20,9 & 16,8 & 23,3 & 23,1 \\
3 & 77,0 & 75,6 & 74,6 & 74,0 & 73,4 & 72,0 & 71,0 & 70,2 & 79,0 & 67,1 & 67,4 \\
\hline L \& L* & & & & & & & & & & & \\
1 & 6,38 & 6,81 & 6,46 & 6,73 & 6,71 & 7,48 & 8,16 & 8,93 & 7,69 & 9,58 & 9,56 \\
2 & 16,6 & 17,6 & 19,0 & 19,3 & 19,9 & 20,5 & 20,8 & 20,9 & 19,8 & 23,3 & 23,1 \\
3 & 46,2 & 48,1 & 48,4 & 50,5 & 50,8 & 49,0 & 46,6 & 48,0 & 48,2 & 48,0 & 46,0 \\
4 & 30,8 & 27,5 & 26,1 & 23,5 & 22,5 & 23,0 & 24,4 & 22,1 & 24,3 & 19,1 & 21,4 \\
\hline
\end{tabular}

* Siguiendo el enfoque de Lindley y Lorgelly (2003) 


\section{Variables socioeconómicas}

Nuestros modelos empíricos tienen la intención de capturar la asociación entre SA y un conjunto de variables socioeconómicas. Hay tres variables representando situación marital (Viudo, Soltero, Div/Sep), con «casado» como la categoría de referencia. Un indicador de etnicidad es introducido (No-Blanco). Cuatro variables binarias han sido construidas para representar el máximo nivel de educación alcanzada, centrándose en el capital humano de los encuestados y basándose en el sistema educativo británico. Estos son: Degree (Grado superior/Primer grado), HND/A (Certificado Nacional, Diploma Nacional, nivel A), O/CSE (nivel O, Certificado de Educación Secundaria). No-Qual (no cualificación) es el caso base de las variables educativas. El tamaño del hogar (HHSize) y el número de niños en el hogar por edad, son también incluidos en el análisis para capturar el momento de vida de los encuestados. La variable de renta es el logaritmo de la renta real equivalente, ajustada utilizando el Índice de Precios de Venta y la escala McClement para ajustar por el tamaño de los hogares y su composición. Se incluye una función polinomial cuártica para la variable edad (Edad, $\left.\operatorname{Edad} 2=\operatorname{Edad}^{2} / 100, \operatorname{Edad} 3=\operatorname{Edad}^{3} / 10,000, \operatorname{Edad} 4=\operatorname{Edad}^{4} / 1,000,000\right)$ y un vector de indicadores anuales para cada período. Las variables están resumidas en la Tabla 3.

TABLA 3

\section{DEFINICIÓN DE LOS REGRESORES}

\begin{tabular}{|ll}
\hline Viudo & 1 si viudo, 0 en otro caso \\
Soltero & 1 si nunca casado, 0 en otro caso \\
Div/Sep & 1 si divorciado o separado, 0 en otro caso \\
No-Blanco & 1 si es miembro de un grupo étnico diferente a blanco, 0 en otro caso \\
Degree & 1 si su cualificación académica más elevada es graduado o graduado supe- \\
& rior, 0 en otro caso \\
HND/A & 1 si la mayor cualificación académica es HND o Nivel A, 0 en otro caso \\
O/CSE & 1 si la mayor cualificación académica es Nivel O o CSE, 0 en otro caso \\
HHSize & Número de personas en el hogar incluyendo al encuestado \\
NCH04 & Número de niños en el hogar con edad comprendida entre 0-4 \\
NCH511 & Número de niños en el hogar con edad comprendida entre 5-11 \\
NCH1218 & Número de niños en el hogar con edad comprendida entre 12-18 \\
Renta & Renta del hogar anual real y equivalente en libras \\
Edad & Edad en años el 1 de Diciembre de la oleada correspondiente
\end{tabular}

Una comprobación de consistencia

Hay una pregunta útil adicional solo disponible en la oleada 9 del Panel de Hogares Británico (incluida en el cuestionario SF-36) sobre la salud del individuo comparada con la de hace un año. A los individuos se les pregunta: «Comparado con hace un año, cómo evaluaría su salud en general ahora?», con 5 posibles respuestas: «mucho mejor», «mejor», «igual», «peor»y «mucho peor». Esta variable une 
las oleadas 8 y 9 y puede ser utilizada para examinar la consistencia de las respuestas obtenidas en la oleada 9 y nos permite centrarnos en el cambio en la identificación de las cinco categorías (en vez de centrarnos en el marco de las preguntas). «Igual» salud comparada con hace un año es percibida por $70,33 \%$ de la muestra, con el siguiente porcentaje más elevado percibiendo un mayor nivel de salud que el año pasado ( $16,22 \%$ de individuos percibieron «mucha mejor»o «mejor» salud) y $13,45 \%$ percibieron «peor» o «mucho peor» salud que hace un año. Si nos centramos solo en estos individuos que percibieron la «misma» salud comparado con el año anterior, podemos analizar la distribución de SA en la oleada 9, dada cada una de las respuestas disponibles en la oleada 8. Los resultados están resumidos en las Tablas 4 y 5 .

La Tabla 4 muestra el porcentaje de individuos que percibieron o bien «excelente»o «buena» salud en la oleada 8 , que evalúan su salud en la oleada 9 como «excelente» o «muy buena» ( si revelaron «excelente» en la oleada 8 ), y «muy buena»o «buena» salud (si percibieron «buena» salud en la oleada 8). Esto proporciona evidencia sobre el efecto de introducir la categoría «muy buena» entre «excelente» y «buena», en la oleada 9. Es posible comprobar los patrones en el ajuste de las respuestas de la oleada 8 a la nueva categoría «muy buena» con respecto a algunas de las variables socioeconómicas, tales como género, el mayor nivel de educación alcanzado, cuantiles de renta y edad. $42,04 \%$ de los individuos que percibieron «excelente» salud en la oleada 8 , evaluaron su salud como «muy buena» cuando esta opción estuvo disponible en la oleada 9 . Este porcentaje es similar para hombres y mujeres $(42,02 \%$ y $42,05 \%$, respectivamente). Por grupos socioeconómicos, se observa que el porcentaje de los individuos que perciben «muy buena» salud en la oleada 9 sigue una tendencia creciente con niveles de educación mayores, i.e. aquéllos que han alcanzado mayor nivel educativo es más probable que cambien su respuesta de la oleada 8 y evalúen su salud como «muy buena» en la oleada 9. Para los cuantiles de renta, aquellos individuos en el cuarto cuantil representan el porcentaje más pequeño de individuos que perciben «muy buena» salud en la oleada 9 . Un gradiente positivo puede verse para los grupos de edades considerados. De hecho, el porcentaje de individuos que perciben «muy buena» salud en la oleada 9 crece mientras los individuos envejecen. El mismo análisis podría ser aplicado a aquellos individuos que revelan «buena» salud en la oleada 8. En este caso, $42,13 \%$ de individuos perciben «muy buena» salud en la oleada 9 . Por género, se observa que más mujeres $(44,13 \%)$ que hombres $(39,73 \%)$ perciben «muy buena» salud en la oleada 9. El porcentaje de aquellos individuos que perciben «muy buena» salud crece con los niveles de educación. Si el grupo más rico de la muestra (Q5) es comparado con el más pobre (Q1), se puede ver que el porcentaje de aquéllos que perciben «muy buena» salud es mayor para aquéllos en el grupo de renta más elevado. Es menos probable que los individuos de mayor edad perciban «muy buena» salud en la oleada 9, una vez que han revelado «buena» salud en la oleada 8.

Los cambios en las respuestas de SA de la oleada 8 a la oleada 9 mostrados en la Tabla 4, no necesariamente implican inconsistencias en las respuestas. Por ejemplo, algunos de los que perciben «excelente» salud en la oleada 8 y consideran que su sa- 
TABLA 4

INTRODUCCIÓN DE LA CATEGORÍA «MUY BUENA» EN LA OLEADA 9

\begin{tabular}{|l|c|c|c|c|}
\hline \multirow{2}{*}{} & \multicolumn{2}{|c|}{ Ex (w8) } & \multicolumn{2}{c|}{ B (w8) } \\
\cline { 2 - 5 } & Ex (w9) & MB (w9) & MB (w9) & B (w9) \\
\hline Todos & 42,12 & 42,04 & 42,13 & 42,48 \\
Hombres & 40,82 & 42,02 & 39,73 & 43,88 \\
Mujeres & 43,51 & 42,05 & 44,13 & 41,32 \\
Degree & 51,37 & 37,70 & 48,85 & 37,38 \\
HND & 40,39 & 44,95 & 43,01 & 42,65 \\
OCSE & 43,51 & 41,08 & 43,67 & 41,39 \\
No-Qual & 35,69 & 43,12 & 37,22 & 45,65 \\
Cuantiles de Renta: & & & & \\
Q1 & 40,68 & 44,07 & 36,96 & 45,54 \\
Q2 & 38,89 & 40,74 & 40,87 & 42,61 \\
Q3 & 39,78 & 45,30 & 43,81 & 41,9 \\
Q4 & 42,86 & 35,29 & 40,69 & 45,41 \\
Q5 & 44,44 & 44,05 & 47,33 & 37,62 \\
Categorías de Edad: & & & & \\
<30 & 41,82 & 38,18 & 43,30 & 37,63 \\
30-50 & 46,84 & 36,92 & 45,75 & 39,20 \\
50-70 & 36,75 & 46,64 & 39,29 & 48,05 \\
70+ & 29,76 & 54,76 & 37,35 & 42,97 \\
\hline
\end{tabular}

lud es todavía la misma, podría n legítimamente percibir «muy buena» salud cuando esta opción está disponible en la oleada 9. Por el contrario, la Tabla 5 proporciona evidencia de inconsistencias de autopercepciones para aquellos individuos que revelan tener el «mismo» nivel de salud en la oleada 9, comparado con el último año. De estos resultados es posible comprobar cómo esta inconsistencia varía entre grupos socioeconómicos. Para este propósito, distinguimos entre aquellos individuos cuyas evaluaciones reflejan o bien una reducción o una mejora en salud, comparando sus respuestas para la oleada 8 con las respuestas proporcionadas en la oleada 9. La etiqueta «reducción» en la Tabla 5 representa a aquellos individuos que llevan a cabo un movimiento inconsistente de una categoría en la oleada 8 a una menor categoría en la oleada 9, siendo inconsistente porque estos individuos revelan tener la «misma» salud en la oleada 9 en comparación con el año pasado. Nosotros asumimos que reducir incluye las posibilidades siguientes: moverse de «excelente» en la oleada 8 a «buena», «regular» o «mala» salud en la oleada 9; moverse de «buena» en la oleada 8 a «regular» o «mala» en la oleada 9; moverse de «regular» en la oleada 8 a «mala» en la oleada 9. Por otra parte, la mejora representa a aquellos individuos que contestan mayores categorías en la oleada 9, habiendo respondido que su salud es la «misma» compara- 
do al año pasado. Estas son las elecciones consideradas: desplazamiento de «buena» en la oleada 8 a «excelente» en la oleada 9; desplazamiento de «regular» en la oleada 8 a «excelente», «muy buena» o «buena» en la oleada 9; desplazamiento de «mala» en la oleada 8 a «excelente», «muy Buena», «buena» o «regular» en la oleada 9; desplazamiento de «muy mala» en la oleada 8 a «mala», «regular», «buena», «muy buena» o «excelente» salud en la oleada 9.

De estos resultados, se puede ver que un mayor porcentaje de individuos que contestaron tener la misma salud en ambas oleadas, mejoran su evaluación de salud $(25,98 \%)$ en la oleada 9 , mientras que $9,88 \%$ perciben una reducción en su propia salud. El porcentaje de mujeres que perciben una mejora es un poco mayor $(23,87 \%)$ que para los hombres $(22,46 \%)$, siendo más probable que la submuestra de hombres sea inconsistente en sus respuestas cuando perciben una reducción en salud (10,33\%). El porcentaje de individuos que perciben una mejora o una reducción en su salud comparado con la oleada 8 decrece con el nivel de educación. Por tanto, los individuos sin cualificaciones tienden a ser menos consistentes. Hay un gradiente negativo con la renta, ya que individuos en el grupo de renta más baja tienden a ser menos consistentes y contestan una mejora en su salud, en comparación con los individuos en los cuantiles más altos. No es posible distinguir un patrón claro de aquéllos que contestan una reducción en salud por los grupos de renta. Sin embargo, si el grupo de renta más bajo (Q1) se compara con el grupo de renta más elevada (Q5), se puede ob-

TABLA 5

EVIDENCIA DE RESPUESTAS INCONSISTENTES

\begin{tabular}{|l|r|r|}
\hline & Reducción & Mejora \\
\hline Todos & $354(9,88 \%)$ & $931(25,98 \%)$ \\
Hombres & $172(10,33 \%)$ & $374(22,46 \%)$ \\
Mujeres & $169(8,81 \%)$ & $458(23,87 \%)$ \\
Degree & $32(6,93 \%)$ & $88(19,04 \%)$ \\
HND & $71(8,33 \%)$ & $181(21,24 \%)$ \\
OCSE & $98(8,85 \%)$ & $246(22,22 \%)$ \\
No-Qual & $139(12,01 \%)$ & $312(26,97 \%)$ \\
Cuantiles de renta: & $51(8,5 \%)$ & $173(28,83 \%)$ \\
Q1 & $66(9,78 \%)$ & $158(23,41 \%)$ \\
Q2 & $57(7,45 \%)$ & $176(23,01 \%)$ \\
Q3 & $67(8,85 \%)$ & $158(20,87 \%)$ \\
Q4 & $46(5,84 \%)$ & $167(21,22 \%)$ \\
Q5 & $30(8,09 \%)$ & \\
Categorías de edad: & $121(7,52 \%)$ & $91(24,53 \%)$ \\
<30 & $89(7,77 \%)$ & $224(21,14 \%)$ \\
$30-50$ & $47(10,24 \%)$ & $139(30,28 \%)$ \\
$50-70$ & & \\
$70+$ &
\end{tabular}


servar que es menos probable que los individuos más ricos perciban una reducción en su salud. No hay un claro patrón tampoco por grupos de edad. Sin embargo, aquéllos mayores de 70 es más probable que revelen una mejora en salud $(30,28 \%)$ o una reducción en la salud (10,24\%), mientras aquéllos de edad entre 50 a 70, es menos probable que sean inconsistentes en sus respuestas, con el 7,77\% percibiendo una reducción en su salud y el 19,55\% percibiendo una mejora en su evaluación de salud.

El análisis de la introducción de «muy buena» salud como categoría de respuesta en la oleada 9, como se muestra en la Tabla 4, indica, en general, que los encuestados son razonablemente consistentes en sus percepciones de SA, dado que afirmaron que su salud había sido la misma entre las oleadas 8 y 9 y que hubieran revelado «excelente» o «buena» salud en la oleada 8 . Sin embargo, cuando se consideran todas las posibles categorías de SA en la oleada 8, hay un grado mayor de inconsistencia en el comportamiento de respuesta entre las oleadas 8 y 9 con hasta el $30 \%$ de los individuos proporcionando una respuesta en la oleada 9 que es inconsistente con la percepción de que su salud se hubiera mantenido igual. Si estamos preparados para asumir que los individuos pueden recordar mejor su estado de salud subyacente hace un año con mayor exactitud con la que recuerdan la categoría de respuesta actual que utilizaron al responder la encuesta del Panel de Hogares Británico, entonces estos resultados proporcionan evidencia de error de medida en la oleada 9 que parece haber sido inducida por el cambio en identificación de las categorías de respuesta de SA cuando pasan al cuestionario SF-36.

\section{Resultados}

\section{El modelo de variable latente}

Los resultados para modelos probit ordenados de SA con 5 categorías, estimados para hombres y mujeres separadamente en todos los datos excluyendo la oleada 9, están respresentados en la Tabla 6. Estos resultados representan una referencia con la que los resultados de incorporar la versión de SA de la oleada 9 perteneciente al cuestionario del SF-36, pueden ser comparados. Un número de características son destacables. Hay un gradiente claro en la relación entre logro educacional y SA con individuos con cualificaciones mayores que perciben mejor salud. También se da la relación positiva esperada entre la renta y salud tanto para hombres como para mujeres.

Observamos una reducción general en la percepción de mejores estados de salud cuando comparamos las distribuciones de SA entre las once oleadas como se muestra en la Figura 1. Aun condicionando por regresores relevantes tales como edad, renta y logro educacional, todavía observamos un gradiente claro entre oleadas, siendo evidente en los coeficientes de los indicadores anuales. Éstos llegan a ser más negativos mientras el cohorte envejece, indicando la percepción de peor salud. Esto podría ser debido a un efecto de edad no enteramente eliminado por el formato de la pregunta de SA o no enteramente controlado al condicionar utilizando un polinomio de edad. Sin embargo, también podría ser debido a los cambios de respuesta mientras los in- 
TABLA 6

MODELOS PROBIT ORDENADOS EXCLUYENDO OLEADA 9

\begin{tabular}{|l|r|r|}
\hline & HOMBRES & MUJERES \\
& NT $=\mathbf{2 , 2 5 3 9}$ & NT $=\mathbf{2 , 8 3 8 6}$ \\
\hline Degree & $0,377(0,058)$ & $0,381(0,052)$ \\
HND/A & $0,260(0,048)$ & $0,279(0,045)$ \\
O/CSE & $0,218(0,049)$ & $0,289(0,041)$ \\
Edad & $0,071(0,060)$ & $0,031(0,059)$ \\
Edad2 & $-0,236(0,193)$ & $-0,123(0,187)$ \\
Edad3 & $0,300(0,262)$ & $0,209(0,249)$ \\
Edad4 & $-0,129(0,126)$ & $-0,126(0,118)$ \\
Ln(renta) & $0,244(0,025)$ & $0,206(0,019)$ \\
yr9293 & $-0,087(0,023)$ & $-0,067(0,020)$ \\
yr9394 & $-0,133(0,024)$ & $-0,095(0,021)$ \\
yr9495 & $-0,170(0,025)$ & $-0,147(0,022)$ \\
yr9596 & $-0,215(0,025)$ & $-0,160(0,023)$ \\
yr9697 & $-0,212(0,027)$ & $-0,202(0,024)$ \\
yr9798 & $-0,237(0,028)$ & $-0,197(0,025)$ \\
yr9899 & $-0,285(0,028)$ & $-0,238(0,025)$ \\
yr9900 & $-0,368(0,029)$ & $-0,336(0,026)$ \\
yr0001 & $-0,336(0,030)$ & $-0,276(0,027)$ \\
yr0102 & 0,747 & 0,117 \\
\hline Cut1 & 1,514 & 0,889 \\
Cut2 & 2,396 & 1,764 \\
Cut3 & 3,736 & 3,119 \\
Cut4 & & $-35.101,4$ \\
Intra-class correlation & $-26.874,1$ & $1,49(0,22)$ \\
\hline Log Likelihood & $0,04(0,85)$ & \\
RESET (p value) & & \\
\hline
\end{tabular}

Notas:

1. SA está basada en la categorización: 1: «muy mala», 2: «mala»; 3 «regular»; 4 «buena»; 5: «excelente» salud.

2. Coeficientes estimados para las variables Viudo, Soltero, Div/Sep, No-Blanco, HHSize, NCH04, NCH511, NCH1218 han sido suprimidos para conservar espacio. Resultados completos están disponibles.

3. Errores estándares entre paréntesis.

dividuos incrementan su edad, reflejando un desplazamiento del índice en el tiempo. Esto implicaría el contexto dentro del cual los individuos evalúan sus propios cambios de salud con el aumento de la edad. Sin embargo esta segunda hipótesis parece improbable ya que la dirección de la tendencia contradice la noción de adaptación: los individuos se adaptan a una peor salud, llegando a ser más optimistas en sus evaluaciones de SA (Groot, 2000). 


\section{El modelo de medida}

La Tabla 7 presenta los resultados de especificaciones de probit ordenados para hombres y mujeres cuando se incorporan los datos de la oleada 9 en SA. Las columnas de las tablas representan las clasificaciones alternativas de SA como se indica en las Tablas 1 y 2 anteriores. Una comparación de los coeficientes de SA con 5 categorías con sus coeficientes respectivos presentados en la Tabla 6 , revela poco cambio en la magnitud de las estimaciones de los efectos de características socioeconómicas en salud, aunque, en general estos efectos son ligeramente superiores a cero. Esto es evidente tanto para hombres como para mujeres. Una comparación adicional de los coeficientes estimados entre las cuatro diferentes categorizaciones de SA revela un grado elevado de consistencia con la excepción de SA con 3 categorías: reducir la variable SA original con cinco categorías no parece afectar sustancialmente la relación estimada entre características socioeconómicas y salud. Este resultado apoya la noción que la especificación del modelo de variable latente subyacente es apropriada. Diferencias mayores en coeficientes entre los cuatro modelos son observados para los puntos de corte estimados indicando, como uno esperaría, que reducir la escala categórica original de cinco puntos a cuatro o tres categorías tiene un mayor efecto en el modelo de medida que determina qué categoría de SA los individuos contestan dada su verdadera salud latente.

\section{Un desplazamiento del índice común}

El hecho de incluir los datos de la oleada 9 tiene un efecto marcado en los coeficientes estimados de los indicadores anuales. Los resultados presentados en la Tabla 6 que excluían la información de la oleada 9, mostró un gradiente decreciente en la salud percibida mientras que el cohorte envejecía. Este gradiente se mantiene tras incluir los datos de la oleada 9. Sin embargo, el coeficiente estimado para el indicador de la oleada 9 (yr9900) en general no se ajusta a la tendencia observada entre los otros años (ver Tabla 7). Esto es particularmente cierto para el modelo de SA 5 donde los coeficientes estimados para yr9900 son sustancialmente mayores que los coeficientes para otros años. Esto no sorprende dado que la construcción y codificación de la variable de SA para la oleada 9 (basada en la versión de SA del cuestionario SF -36) es diferente a la construcción y codificación de la variable SA en otras oleadas. El coeficiente estimado elevado y negativo del indicador anual para la oleada 9 aparece como un artefacto directo de la forma en la cual la variable ha sido construida.

Si consideramos los coeficientes de la oleada 9 para los modelos que adoptan la categorización de SA 4 y el enfoque basado en la metodología de Lindley y Lorgelly ( $\mathrm{L} \& \mathrm{~L}$ ), entonces podemos ver que para la submuestra de hombres, los estimadores están mas cerca de ser consistentes con las tendencias en los gradientes observados entre los otros años. Sin embargo, los estimadores están más cerca de cero que la consistencia verdadera que las tendencias temporales predicirían. Para las mujeres, los estimadores no se ajustan a las tendencias observadas entre otros años y están mucho más cerca de cero que lo que se habría predicho en base a las tendencias. El valor absoluto más pequeño del coeficiente de la oleada 9 obtenido tanto para hombres como 
TABLA 7

MODELOS PROBIT ORDENADOS, INCLUYENDO LOS DATOS DE LA OLEADA 9

\begin{tabular}{|c|c|c|c|c|c|c|c|c|}
\hline & \multicolumn{4}{|c|}{$\begin{array}{c}\text { Hombres } \\
\text { NT }=24791\end{array}$} & \multicolumn{4}{|c|}{$\begin{array}{c}\text { Mujeres } \\
\text { NT }=\mathbf{3 1 2 2 5}\end{array}$} \\
\hline & SA 5 & SA 4 & SA 3 & $L \& L$ & SA 5 & SA 4 & SA 3 & $\mathrm{~L} \& \mathrm{~L}$ \\
\hline Degree & $0,385(0,058)$ & $0,381(0,058)$ & $0,454(0,068)$ & $0,377(0,057)$ & $0,388(0,053)$ & $0,382(0,052)$ & $0,477(0,060)$ & $0,383(0,052)$ \\
\hline HND/A & $0,266(0,047)$ & $0,262(0,048)$ & $0,324(0,054)$ & $0,263(0,047)$ & $0,287(0,045)$ & $0,280(0,045)$ & $0,338(0,051)$ & $0,286(0,045)$ \\
\hline 0/CSE & $0,222(0,048)$ & $0,219(0,049)$ & $0,268(0,055)$ & $0,219(0,048)$ & $0,292(0,041)$ & $0,289(0,041)$ & $0,326(0,045)$ & $0,290(0,040)$ \\
\hline Edad & $0,080(0,059)$ & $0,069(0,060)$ & $0,086(0,070)$ & $0,072(0,059)$ & $0,043(0,059)$ & $0,044(0,060)$ & $0,035(0,066)$ & $0,038(0,059)$ \\
\hline Edad2 & $-0,266(0,192)$ & $-0,229(0,192)$ & $-0,287(0,226)$ & $-0,234(0,190)$ & $-0,163(0,186)$ & $-0,163(0,189)$ & $-0,140(0,207)$ & $-0,143(0,187)$ \\
\hline Edad3 & $0,341(0,259)$ & $0,288(0,259)$ & $0,359(0,304)$ & $0,294(0,246)$ & $0,264(0,246)$ & $0,259(0,250)$ & $0,244(0,274)$ & $0,233(0,248)$ \\
\hline Edad4 & $-0,148(0,125)$ & $-0,122(0,124)$ & $-0,147(0,144)$ & $-0,124(0,123)$ & $-0,152(0,116)$ & $-0,148(0,118)$ & $-0,148(0,129)$ & $-0,136(0,118)$ \\
\hline Ln(renta) & $0,236(0,025)$ & $0,241(0,025)$ & $0,274(0,030)$ & $0,238(0,025)$ & $0,207(0,019)$ & $0,207(0,019)$ & $0,212(0,021)$ & $0,208(0,019)$ \\
\hline yr 9293 & $-0,085(0,022)$ & $-0,091(0,023)$ & $-0,070(0,030)$ & $-0,089(0,022)$ & $-0,067(0,020)$ & $-0,071(0,020)$ & $-0,037(0,024)$ & $-0,070(0,020)$ \\
\hline yr9394 & $-0,130(0,024)$ & $-0,139(0,024)$ & $-0,121(0,031)$ & $-0,136(0,024)$ & $-0,094(0,021)$ & $-0,097(0,022)$ & $-0,043(0,026)$ & $-0,096(0,022)$ \\
\hline yr9495 & $-0,167(0,025)$ & $-0,179(0,026)$ & $-0,136(0,033)$ & $-0,175(0,025)$ & $-0,146(0,022)$ & $-0,152(0,023)$ & $-0,057(0,027)$ & $-0,149(0,022)$ \\
\hline yr9596 & $-0,210(0,025)$ & $-0,222(0,026)$ & $-0,145(0,034)$ & $-0,217(0,025)$ & $-0,158(0,023)$ & $-0,164(0,024)$ & $-0,088(0,028)$ & $-0,161(0,023)$ \\
\hline yr9697 & $-0,207(0,027)$ & $-0,221(0,028)$ & $-0,196(0,035)$ & $-0,216(0,027)$ & $-0,200(0,023)$ & $-0,207(0,024)$ & $-0,143(0,028)$ & $-0,203(0,024)$ \\
\hline yr9798 & $-0,232(0,027)$ & $-0,238(0,028)$ & $-0,240(0,036)$ & $-0,233(0,027)$ & $-0,195(0,025)$ & $-0,196(0,025)$ & $-0,173(0,029)$ & $-0,193(0,025)$ \\
\hline yr9899 & $-0,280(0,028)$ & $-0,294(0,029)$ & $-0,278(0,036)$ & $-0,287(0,028)$ & $-0,235(0,025)$ & $-0,242(0,025)$ & $-0,194(0,029)$ & $-0,238(0,025)$ \\
\hline yr9900 & $-0,833(0,030)$ & $-0,288(0,027)$ & $0,183(0,038)$ & $-0,242(0,029)$ & $-0,745(0,026)$ & $-0,164(0,024)$ & $0,348(0,032)$ & $-0,128(0,026)$ \\
\hline yr0001 & $-0,361(0,029)$ & $-0,374(0,029)$ & $-0,356(0,035)$ & $-0,365(0,029)$ & $-0,332(0,025)$ & $-0,340(0,026)$ & $-0,274(0,030)$ & $-0,333(0,026)$ \\
\hline yr0102 & $-0,329(0,029)$ & $-0,344(0,030)$ & $-0,346(0,036)$ & $-0,335(0,030)$ & $-0,272(0,026)$ & $-0,280(0,027)$ & $-0,257(0,031)$ & $-0,275(0,027)$ \\
\hline Cutl & 0,718 & 1,418 & 1,976 & 1,458 & 0,22 & 1,005 & 1,086 & 0,999 \\
\hline Cut2 & 1,530 & 2,306 & 2,892 & 2,339 & 1,028 & 1,884 & 1,992 & 1,874 \\
\hline Cut3 & 2,423 & 3,696 & & 3,681 & 1,915 & 3,291 & & 3,228 \\
\hline Cut4 & 3,733 & & & 3,245 & & & & \\
\hline Log Likelihood & $-30.074,2$ & $-28.436,4$ & $-16.197,9$ & $-28.797,5$ & $-39.110,8$ & $-36.730,8$ & $-23.046,3$ & $-37.223,2$ \\
\hline RESET (p value) & $0,29(0,59)$ & $0,00(0,98)$ & $0,03(0,87)$ & $0,02(0,88)$ & $0,17(0,68)$ & $2,35(0,13)$ & $3,35(0,07)$ & $2,92(0,09)$ \\
\hline
\end{tabular}

Notes:

1. Coeficientes estimados para las variables Viudo, Soltero, Div/Sep, No-Blanco, HHSize, NCH04, NCH511, NCH1218 han sido suprimidas para conservar espacio. Resultados completos están disponibles.

2. Los errores estándares entre paréntesis.

para mujeres parecería reflejar dos cosas: primero, un «efecto suelo» en la oleada 9 para la categoría menor de salud («mala») y en segundo lugar, la mayor proporción de encuestados valorando su salud en la categoría combinada de «buena, muy buena» salud comparado con la proporción en la categoría equivalente de «buena» salud en las otras oleadas. Las diferencias en las proporciones pueden ser vistas en la Ta- 
bla 2. El efecto combinado induciría un desplazamiento del índice hacia la percepción de mejor estado de salud en la oleada 9.

El coeficiente estimado para la oleada 9 cuando se considera la categorización de SA 3 es positivo para hombres y elevado y positivo para mujeres. Esto no se ajusta a las tendencias observadas en los coeficientes estimados entre datos de otros años. Un vistazo rápido a la Tabla 2 muestra que la distribución de respuestas en la oleada 9 cuando se utiliza la categorización de SA 3, está sesgada hacia la percepción de mejor estado de salud. De nuevo, esto es consistente con un desplazamiento del índice hacia la percepción de mejor salud en la oleada 9.

\section{Sesgo de respuesta}

Los resultados mostrados anteriormente indican que los datos son consistentes con un desplazamiento del índice en la oleada 9. Para evaluar si el desplazamiento observado en los puntos de corte están sistemáticamente relacionados con las características del subgrupo de la población, incluimos interacciones del indicador para la oleada 9 con las variables socioeconómicas. La Tabla 8 presenta los resultados para las interacciones con logro educacional, renta y edad. De nuevo, los resultados se presentan para especificaciones de probit ordenados solamente. Los modelos fueron estimados para la variable original de SA con cinco categorías junto con la categorización de SA 4 y el enfoque sugerido por Lindley y Lorgelly. Los tests del ratio de la verosimilitud logarítmica de modelos restringidos (sin términos de interacción) frente a modelos no restringidos (con términos de interacción) rechazan el modelo restringido para la variable SA con cinco categorías y la categorización de Lindley y Lorgelly de SA para las mujeres solamente. Sin embargo, una comparación de los coeficientes individuales con sus errores estándares revela términos de interacción no significativos entre las variables socioeconómicas y el indicador para la oleada 9, para cualquiera de las especificaciones comprobadas del modelo ${ }^{7}$. Este último resultado sugiere que los puntos de corte no están sistemáticamente relacionados con subgrupos de población identificables.

\section{Desplazamiento del punto de corte}

En general no es posible distinguir entre el desplazamiento de puntos de corte y heterogeneidad en la ecuación de la variable latente. Sin embargo, si se asume que las ecuaciones (1) y (9) están correctamente especificadas — esto es, no hay heterogeneidad en las $\beta$ 's y el sesgo de respuesta lleva al desplazamiento de los puntos de corte- entonces los resultados del POG pueden ser interpretados como separador de los efectos de los regresores en salud verdadera y sesgo de respuesta. Los anteriores son capturados por las $\beta$ 's y los últimos, por las $\delta$ 's. Para interpretar los resultados de los estimadores del modelo POG, es necesario tener en cuenta que el punto de corte superior $\left(\mu_{4}^{0}\right)$ está fijo. Dos versiones del modelo POG son estimadas. En la primera versión, los puntos de corte son modelizados como una función de las variables explicativas correspondientes a la ecuación (7). Éste es el modelo POG restringido. La

\footnotetext{
7 Esto es también verdadero para los términos de interacción no presentados en la Tabla 8.
} 


\section{TABLA 8}

MODELOS PROBIT ORDENADOS CON INTERACCIONES, INCLUYENDO LOS DATOS DE LA OLEADA 9

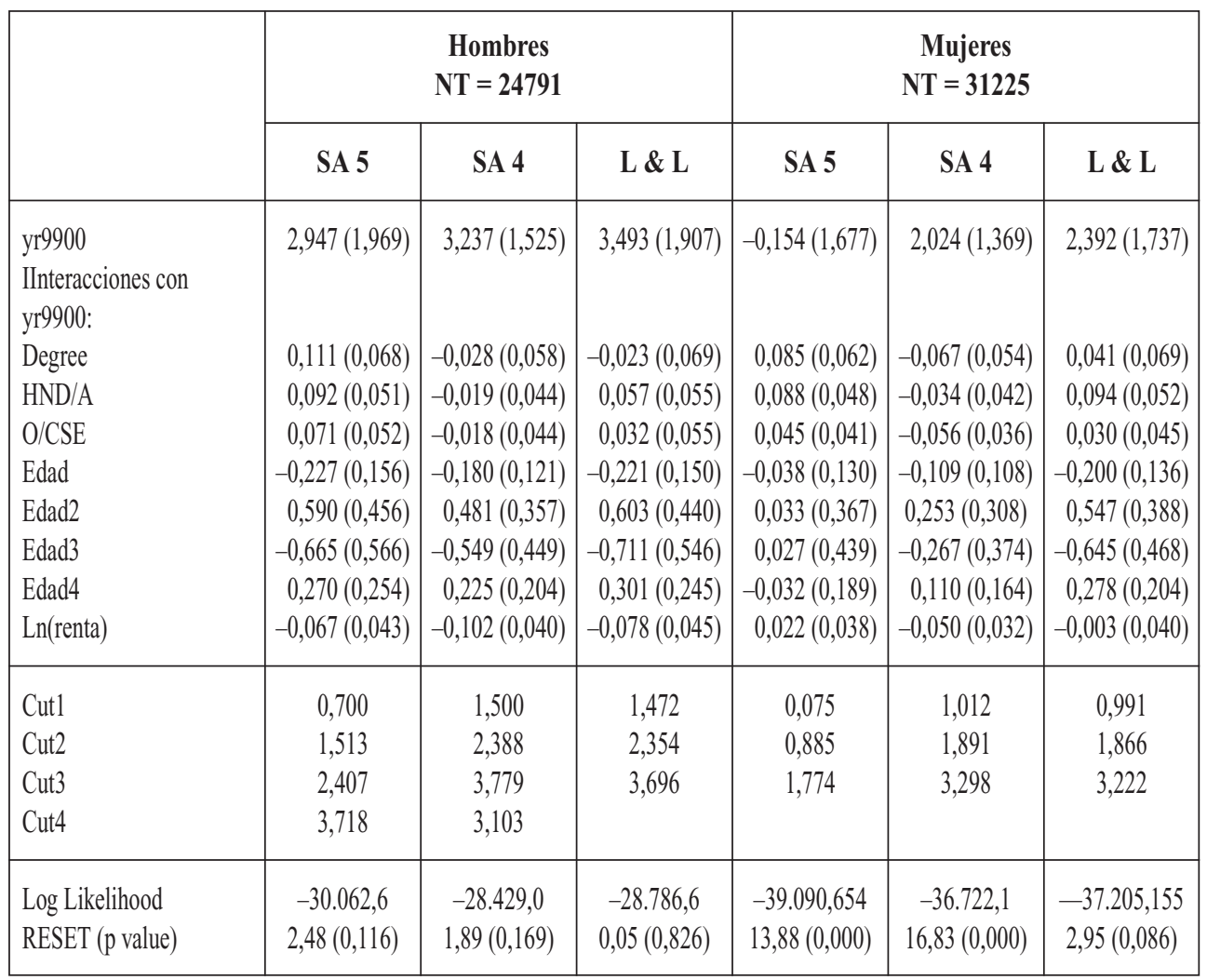

Notas:

1. Coeficientes estimados para las variables Viudo, Soltero, Div/Sep, No-Blanco, HHSize, NCH04, NCH511, NCH1218 y sus interacciones con el indicador de la oleada 9 han sido suprimidos para conservar espacio.

2. Errores estándares entre paréntesis.

segunda versión del modelo POG amplía el grupo de variables explicativas utilizadas para comprobar la constancia de los umbrales para los términos de interacción entre el conjunto inicial de variables explicativas utilizadas en el modelo restrictivo y el indicador de la oleada 9. A este modelo lo llamamos modelo POG no restringido. El modelo no restringido corresponde a las ecuaciones (7) y (8).

La Tabla 9 muestra los resultados para el modelo POG restringido para hombres y mujeres encuestados, en concreto, los coeficientes obtenidos para las variables educativas y para el indicador de la oleada 9 . Se puede observar que para las variables de educación y renta los coeficientes correspondientes en el módelo de índice lineal presenta el signo positivo esperado, para ambos hombres y mujeres. Las magnitudes de los $\beta$ 's son similares pero tienden a ser más pequeños en los modelos POG compara- 
dos al probit ordenado estándar. Cuando se permite que los umbrales varíen con las variables explicativas, los coeficientes $\delta_{j}^{0}$ son negativos. Este resultado implica que, mientras los encuestados incrementan o bien el nivel de educación o bien el nivel de su renta, su evaluación de salud mejora. Entonces, los individuos con mayor renta o nivel de educación tienden a ser más optimistas sobre su salud.

Comparando los coeficientes correspondientes al nivel de educación para hombres, se puede ver que el valor absoluto de los coeficientes $\delta_{j}^{0}$ decrece con el nivel de educación, implicando que los individuos con mayor nivel educativo son más optimistas sobre su salud que aquéllos con niveles inferiores de educación alcanzados. Los mismos resultados se aplican a los coeficientes para la variable de renta. Resultados similares se encuentran para la submuestra de mujeres. Los coeficientes para el nivel de educación y variables de renta tienen el signo esperado. Sin embargo, en términos absolutos, se puede observar que los coeficientes son menores para la mujeres que para los hombres. Entonces, el desplazamiento en los puntos de corte es menor para las mujeres.

La Tabla 10 presenta los resultados para POG no restringido. En este caso todos los términos de interacción entre el grupo de variables explicativas y el indicador para la oleada 9 son utilizados para modelizar tanto el índice de salud latente como los umbrales de los puntos de corte. Se puede ver que los coeficientes estimados para los principales efectos de logro educacional y renta son muy similares a los resultados obtenidos en el modelo restringido. Esto se mantiene para ambos hombres y mujeres. Sin embargo, el coeficiente estimado para el indicador de oleada 9 difiere dramáticamente y para hombres refleja más cerca los obtenidos por el modelo probit ordenado incluyendo términos de interacción, presentados en la Tabla 8.

Solo un número pequeño de los parámetros adicionales estimados en el modelo POG no restringido logran ser significativos estadisticamente al nivel 5\%. De los restantes 48 parámetros estimados (adicionales al modelo restringido), 3 lograron se significativos estadísticamente para mujeres y solo 1 para hombres ${ }^{8}$. Un test de ratio de verosimilitud logarítmica del modelo no restringido frente al modelo restringido no rechaza la hipótesis nula de que las restricciones son válidas, sugiriendo que el modelo POG restringido es una especificación adecuada para estos datos ${ }^{9}$. De nuevo, esto sugiere que el cambio en la frase de la variable SA en la oleada 9 no está asociada con ningún desplazamiento de los puntos de corte en el modelo de medida.

\section{Índices de Concentración y de Movilidad}

Para completar este análisis, presentamos estimadores de desigualdad en salud relacionada con la renta y movilidad (van Doorslaer y Koolman, 2004, Jones y López, 2004) basados en las diferentes medidas de SA. En la Tabla 11, índices de con-

8 Para la submuestra de mujeres éstos son: yr9900x HND/A, yr9900x O/CSE y yr9900x Div/Sep como parámetros para el punto de corte 3 (Cut 3). Para hombres, yr9900x HND/A para el punto de corte 3 es significativo.

${ }_{9}$ Para los hombres: $\chi_{47}^{2}=42,2 ; p=0,67$, para las mujeres: $\chi_{48}^{2}=57,8 ; p=0,16$. 
TABLA 9

PROBIT ORDENADO GENERALIZADO (POG) - MODELOS RESTRINGIDOS

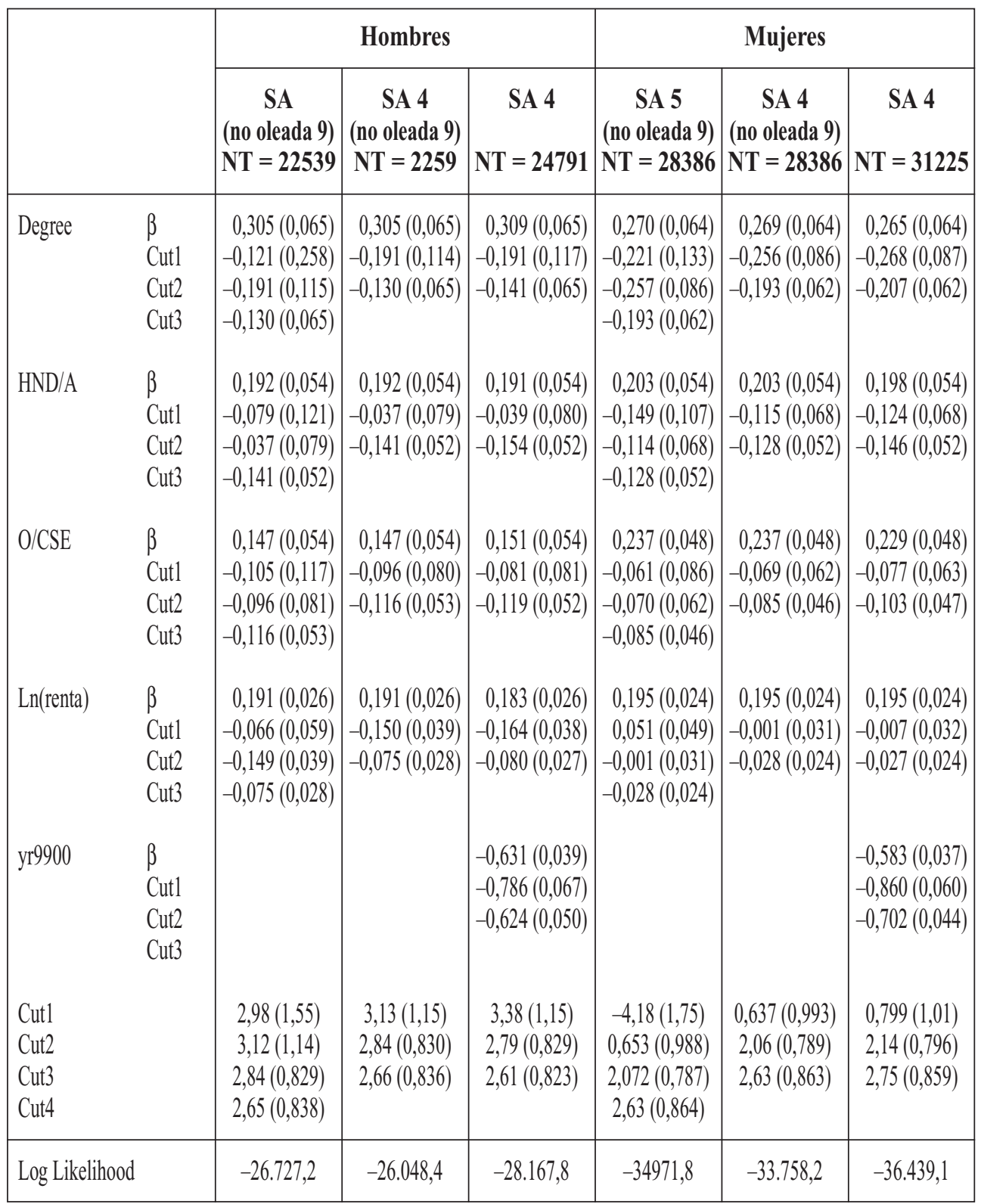

Notas:

1. Coeficientes estimados para las variables Viudo, Soltero, Div/Sep, No-Blanco, HHSize, NCH04, NCH511, NCH1218 y sus interacciones con el indicador de la oleada 9 han sido suprimidos para conservar espacio.

2. Errores estándares entre paréntesis. 
TABLA 10

PROBIT ORDENADO GENERALIZADO (POG) - MODELOS NO RESTRINGIDOS

\begin{tabular}{|c|c|c|c|}
\hline & & $\begin{array}{c}\text { Hombres } \\
\text { SA } 4 \\
\text { NT = 24791 }\end{array}$ & $\begin{array}{c}\text { Mujeres } \\
\text { SA } 4 \\
\text { NT }=31225\end{array}$ \\
\hline Degree & $\begin{array}{l}\beta \\
\text { Cut1 } \\
\text { Cut2 }\end{array}$ & $\begin{array}{r}0,308(0,066) \\
-0,183(0,115) \\
-0,127(0,065)\end{array}$ & $\begin{array}{r}0,270(0,065) \\
-0,256(0,087) \\
-0,193(0,063)\end{array}$ \\
\hline $\mathrm{HND} / \mathrm{A}$ & $\begin{array}{l}\beta \\
\text { Cut1 } \\
\text { Cut2 }\end{array}$ & $\begin{array}{r}0,194(0,054) \\
-0,033(0,080) \\
-0,140(0,052)\end{array}$ & $\begin{array}{r}0,204(0,055) \\
-0,115(0,068) \\
-0,129(0,053)\end{array}$ \\
\hline $\mathrm{O} / \mathrm{CSE}$ & $\begin{array}{l}\beta \\
\text { Cut1 } \\
\text { Cut2 }\end{array}$ & $\begin{array}{r}0,149(0,054) \\
-0,093(0,081) \\
-0,115(0,053)\end{array}$ & $\begin{array}{r}0,238(0,049) \\
-0,069(0,063) \\
-0,086(0,047)\end{array}$ \\
\hline Ln(renta) & $\begin{array}{l}\beta \\
\text { Cut1 } \\
\text { Cut2 }\end{array}$ & $\begin{array}{c}0,188(0,027) \\
-0,165(039) \\
-0,081(0,028)\end{array}$ & $\begin{array}{c}0,196(0,025) \\
-0,002(032) \\
-0,029(0,024)\end{array}$ \\
\hline Yr9900 & $\begin{array}{l}\beta \\
\text { Cut1 } \\
\text { Cut2 }\end{array}$ & $\begin{array}{r}2,365(3,043) \\
1,394(6,457) \\
-4,010(3,972)\end{array}$ & $\begin{array}{r}0,283(2,697) \\
-0,461(4,878) \\
-3,315(3,561)\end{array}$ \\
\hline \multicolumn{4}{|c|}{ Interacciones con yr9900: } \\
\hline Degree & $\begin{array}{l}\beta \\
\text { Cut1 } \\
\text { Cut2 }\end{array}$ & $\begin{array}{r}0,002(0,104) \\
-0,223(0,251) \\
-0,208(0,142)\end{array}$ & $\begin{array}{l}-0,080(0,101) \\
-0,196(0,235) \\
-0,227(0,138)\end{array}$ \\
\hline $\mathrm{HND} / \mathrm{A}$ & $\begin{array}{l}\beta \\
\text { Cut1 } \\
\text { Cut2 }\end{array}$ & $\begin{array}{l}-0,052(0,087) \\
-0,106(0,141) \\
-0,226(0,109)\end{array}$ & $\begin{array}{l}-0,109(0,084) \\
-0,124(0,153) \\
-0,281(0,107)\end{array}$ \\
\hline $\mathrm{O} / \mathrm{CSE}$ & $\begin{array}{l}\beta \\
\text { Cut1 } \\
\text { Cut2 }\end{array}$ & $\begin{array}{c}0,030(0,088) \\
0,208(139) \\
-0,060(0,108)\end{array}$ & $\begin{array}{c}-0,140(0,076) \\
-0,120(123) \\
-0,289(0,094)\end{array}$ \\
\hline Ln(renta) & $\begin{array}{l}\beta \\
\text { Cut1 } \\
\text { Cut2 }\end{array}$ & $\begin{array}{c}-0,055(0,053) \\
{ }^{\dagger} \\
-0,003(0,066)\end{array}$ & $\begin{array}{r}0,002(0,051) \\
-0,118(0,091) \\
0,021(0,066)\end{array}$ \\
\hline $\begin{array}{l}\text { Cut1 } \\
\text { Cut2 } \\
\text { Cut3 }\end{array}$ & & $\begin{array}{l}3,204(1,153) \\
2,856(0,830) \\
2,622(0,835)\end{array}$ & $\begin{array}{c}0,639(0,994) \\
2,069(0,789) \\
2,636(0,864)\end{array}$ \\
\hline Log Likelihood & & $-28.146,7$ & $-36.410,2$ \\
\hline
\end{tabular}

Notas:

1. Coeficientes estimados para las variables Viudo, Soltero, Div/Sep, No-Blanco, HHSize, NCH04, NCH511, NCH1218 y sus interacciones con el indicador de la oleada 9 han sido suprimidos para conservar espacio.

2. Errores estándares entre paréntesis.

3. † Los estimadores de máxima verosimilitud en este modelo sufrieron problemas de optimización. Una forma empírica de solucionarlo se logró imponiendo la restricción que el coeficiente de la variable yr9900xLn(Renta) para el segundo punto de corte era igual a cero. 
centración a corto y largo plazo son presentados, junto con índices de movilidad para salud autopercibida para todos los individuos. Dado el cambio en la pregunta de la variable SA en la oleada 9, hemos utilizado medidas de SA con 5, 4 y 3 categorías para todas las oleadas como se describe en la Tabla 1. El enfoque de Lindley y Lorgelly también ha sido aplicado para obtener la variable de SA con 4 categorías para todas las oleadas. La medida de renta utilizada para construir los índices es la renta real equivalente que ha sido ajustada utilizando el Índice de Precios de Venta y la escala McClement para ajustar por el tamaño del hogar y su composición. Se utiliza un panel equilibrado, en el que solo los individuos de la primera oleada que fueron entrevistados en cada siguiente oleada son incluidos. Siguiendo el ejemplo de Contoyannis et al. (2003), incluimos individuos que proporcionaron una entrevista completa en cada oleada.

En la Tabla 11, se observa que los índices de concentración en el corto plazo para todas las oleadas son positivos, indicando que hay desigualdad en salud que favorece a los más ricos en todos los períodos. También es posible ver un incremento en el tamaño de los índices de concentración a corto plazo en el tiempo. Sin embargo, esta tendencia se rompe en la oleada 9 donde todas las construcciones de SA son consideradas, con la excepción de la variable SA construida siguiendo el enfoque propuesto por Lindley y Lorgelly (columna titulada L\&L). En concreto, para SA con 5 categorías hay un desplazamiento hacia una posición superior en el índice de concentración a corto plazo para la oleada 9. Para SA con 3 y 4 categorías, el estimador para la oleada 9 se desplaza hacia abajo. Los índices de concentración a largo plazo también se

TABLA 11

RESULTADOS PARA LOS ÍNDICES DE CONCENTRACIÓN E ÍNDICE DE MOVILIDAD - TODOS LOS INDIVIDUOS

\begin{tabular}{|c|c|c|c|c|}
\hline & SA 5 & SA 4 & $\mathbf{L} \& \mathbf{L}$ & SA 3 \\
\hline \multicolumn{5}{|l|}{ IC corto plazo } \\
\hline oleada 1 & 0,020 & 0,025 & 0,025 & 0,017 \\
\hline oleada 2 & 0,021 & 0,027 & 0,027 & 0,019 \\
\hline oleada 3 & 0,023 & 0,030 & 0,030 & 0,020 \\
\hline oleada 4 & 0,024 & 0,031 & 0,031 & 0,023 \\
\hline oleada 5 & 0,025 & 0,032 & 0,032 & 0,022 \\
\hline oleada 6 & 0,025 & 0,032 & 0,032 & 0,023 \\
\hline oleada 7 & 0,027 & 0,034 & 0,034 & 0,026 \\
\hline oleada 8 & 0,028 & 0,035 & 0,035 & 0,026 \\
\hline oleada 9 & 0,039 & 0,028 & 0,035 & 0,023 \\
\hline oleada 10 & 0,029 & 0,037 & 0,037 & 0,028 \\
\hline oleada 11 & 0,029 & 0,038 & 0,038 & 0,028 \\
\hline IC largo plazo & 0,030 & 0,036 & 0,037 & 0,026 \\
\hline Índice de Movilidad & $-0,135$ & $-0,134$ & $-0,133$ & $-0,129$ \\
\hline
\end{tabular}


muestran, siendo positivos para todas las variables consideradas. El valor negativo del índice de movilidad implica que el nivel de desigualdad en salud relacionado con la renta en el largo plazo es mayor que lo que podríamos inferir de la información procedente de secciones cruzadas. Si comparamos los estimadores entre construcciones de SA, es posible ver que los índices de concentración a corto y largo plazo junto con los índices de movilidad, son muy similares entre SA con 4 categorías y el enfoque propuesto por L\&L. Sin embargo, los estimadores para SA con 5 o 3 categorías son inferiores que para el resto de construcciones.

Las Tablas 12 y 13 muestran los resultados de los índices de concentración e índice de movilidad, diferenciando entre encuestados hombres y mujeres. El índice de concentración a corto plazo para hombres y mujeres es positivo, indicando que hay desigualdad en salud que favorece a los más ricos en todos los períodos. Hay también un aumento en el tamaño de los estimadores en el tiempo. Aunque los hombres comienzan con un menor índice de concentración que las mujeres en la oleada 1 , sus estimadores alcanzan mayores valores en las últimas oleadas del panel, comparado con las mujeres. Este resultado se aplica a las construcciones de SA que han sido utilizadas. Considerando los resultados por género, puede ser visto que la tendencia creciente de los índices de concentración a corto plazo en el tiempo también se rompe en la oleada 9; siendo el desplazamiento evidente para todas las construcciones de SA, con la excepción del enfoque de L\&L. De nuevo, podemos ver un desplazamiento hacia posiciones superiores en la oleada 9 para la variable SA con 5 categorías y un desplazamiento hacia abajo para SA con 4 y 3 categorías. Esto se aplica tanto para hombres como para mujeres. El índice de concentración a largo plazo es también positivo para hombres y mujeres, siendo más elevados para mujeres que para hombres. Estos estimadores son mayores para SA con 4 categorías y bajo el enfoque L\&L que para el resto de construcciones de SA.

\section{Conclusión}

Varios índices e instrumentos están disponibles para obtener información válida sobre el nivel general de salud del individuo. La fiabilidad de la información subjetiva tal como una medida general de estado de salud autopercibida plantea preocupaciones debido a ser vulnerable a diversos factores de sesgo (Knäuper y Turner, 2000). Sin embargo, medidas objetivas del estado de salud no son frecuentes en datos de encuestas y si existen, suelen ser demasiado específicas a condiciones de salud particulares. Además, su aplicabilidad como una medida general del estado de salud del individuo está a menudo limitada. Un atractivo de las medidas de estado de salud general es su habilidad para encapsular y resumir una multitud de condiciones de salud, pero tienen el coste de estar ampliamente basadas en evaluaciones subjetivas y de autopercepción. Este es el caso en el Panel de Hogares Británico, que ofrece una fuente rica y diversa de información sobre el comportamiento individual y la situación socioeconómica. El tema que rodea el uso de estado de salud general autopercibido está 
TABLA 12

RESULTADOS PARA LOS ÍNDICES DE CONCENTRACIÓN E ÍNDICE DE MOVILIDAD - HOMBRES

\begin{tabular}{|r|r|r|r|r|}
\hline & SA 5 & SA 4 & L\&L & SA 3 \\
\hline IC Corto plazo & & & & \\
oleada 1 & 0,016 & 0,019 & 0,019 & 0,014 \\
oleada 2 & 0,017 & 0,022 & 0,022 & 0,015 \\
oleada 3 & 0,020 & 0,026 & 0,026 & 0,017 \\
oleada 4 & 0,023 & 0,030 & 0,030 & 0,025 \\
oleada 5 & 0,024 & 0,031 & 0,031 & 0,020 \\
oleada 6 & 0,024 & 0,031 & 0,031 & 0,022 \\
oleada 7 & 0,026 & 0,033 & 0,033 & 0,026 \\
oleada 8 & 0,027 & 0,034 & 0,034 & 0,026 \\
oleada 9 & 0,034 & 0,026 & 0,032 & 0,022 \\
oleada 10 & 0,031 & 0,039 & 0,039 & 0,030 \\
oleada 11 & 0,030 & 0,039 & 0,039 & 0,029 \\
\hline IC largo plazo & 0,028 & 0,346 & 0,035 & 0,025 \\
Índice de Movilidad & $-0,131$ & $-0,133$ & $-0,130$ & $-0,122$ \\
\hline
\end{tabular}

TABLA 13

RESULTADOS PARA LOS ÍNDICES DE CONCENTRACIÓN E ÍNDICE DE MOVILIDAD - MUJERES

\begin{tabular}{|r|r|r|r|r|}
\hline & SAH 5 & SAH 4 & L\&L & SAH 3 \\
\hline IC Corto plazo & & & & \\
oleada 1 & 0,022 & 0,028 & 0,028 & 0,0200 \\
oleada 2 & 0,023 & 0,029 & 0,029 & 0,021 \\
oleada 3 & 0,024 & 0,031 & 0,031 & 0,021 \\
oleada 4 & 0,023 & 0,030 & 0,030 & 0,021 \\
oleada 5 & 0,024 & 0,032 & 0,032 & 0,022 \\
oleada 6 & 0,024 & 0,031 & 0,031 & 0,023 \\
oleada 7 & 0,026 & 0,033 & 0,003 & 0,024 \\
oleada 8 & 0,027 & 0,031 & 0,034 & 0,026 \\
oleada 9 & 0,041 & 0,029 & 0,035 & 0,022 \\
oleada 10 & 0,026 & 0,033 & 0,033 & 0,025 \\
oleada 11 & 0,027 & 0,035 & 0,035 & 0,027 \\
\hline IC largo plazo & 0,029 & 0,036 & 0,036 & 0,026 \\
Índice de Movilidad & $-0,141$ & $-0,136$ & $-0,138$ & $-0,136$ \\
\hline
\end{tabular}


adicionalmente interferido en la BHPS debido al cambio en la pregunta de SA en la oleada 9. La incorporación del cuestionario SF-36 en la oleada nueve y el consecuente cambio en la frase de la pregunta de SA junto con un cambio en las categorías de respuestas disponibles, plantean desafíos para el analista que desea llevar a cabo trabajo longitudinal donde el centro de interés es el estado de salud. Sin embargo, e importante, el cambio en la pregunta de SA proporciona una oportunidad para investigar el grado y naturaleza de sesgo de respuesta y más específicamente si grupos de individuos, identificados a través de características socioeconómicas, perciben de diferente forma el cambio en el instrumento de medida. Si este es el caso, entonces los resultados tienen implicaciones para modelizar SA en la BHPS y más generalmente. Evidencia de sesgo de respuesta en la forma de desplazamiento de punto de corte sugeriría que los modelos probit ordenados estándares de SA son excesivamente restringidos y limitan la estimación flexible del efecto de los regresores en las probabilidades de resultados.

Se ha dedicado considerable atención a la fiabilidad de SA y el grado de contaminación por error de medida; el cambio en la frase en la oleada 9 proporciona una forma de experimento natural que nos permite evaluar la sensibilidad del análisis de datos de panel a un cambio en el instrumento de medida. Primero, mostramos el efecto que el cambio de frase de la pregunta SA tiene en los modelos longitudinales de SA y en segundo lugar, cómo esta información puede ser explotada para informar sobre la naturaleza de sesgo de respuesta. Progresivamente, especificaciones más generales de sesgo de respuesta son aplicadas utilizando modelos probit ordenados estándares y generalizados para datos de panel. Éstos están diseñados específicamente para comprobar las hipótesis sobre el papel de la heterogeneidad y sesgo de respuesta en las respuestas de los individuos a la pregunta de SA. Adicionalmente, dado el creciente uso de SA en estudios de desigualdades en salud, exploramos la sensibilidad del índice de concentración de la desigualdad en salud y de movilidad en salud a cambios en la medida de SA.

Nuestros resultados sugieren que reducir la variable original de SA para crear una categorización que tiene un soporte común sobre las dos versiones de la pregunta no afecta sustancialmente a la relación estimada entre características socioeconómicas y salud autopercibida. La construcción de la nueva SA se consigue mejor reduciendo la variable de SA a una versión con 4 categorías, bien combinando «muy mala» $\mathrm{y}$ «mala» salud en las oleadas 1-8 y 10-11 y «buena» y «muy buena» en la oleada 9 o adoptando la metodología propuesta por Lindley y Lorgelly (2003). A nivel práctico, esto es útil para estudios longitudinales del Panel de Hogares Británico u otros datos de panel que incorporen un cambio en la pregunta de SA, que desean hacer uso completo de todos los datos disponibles, pero también es relevante para estudios comparativos que se basen en fuentes de datos con versiones de la pregunta de salud autopercibida.

La hipótesis que nuestro modelo de variable latente subyacente de SA es una especificación apropiada, es apoyada por el resultado de que reducir las categorías de SA no altera sustancialmente la relación entre características socioeconómicas y salud. Basándonos en este resultado, nuestras investigaciones de sesgo de respuesta su- 
gieren que mientras hay evidencia de un desplazamiento paralelo del índice en el uso de umbrales por parte de los encuestados al ajustar el estado de salud subyacente con las categorías de SA, la magnitud de este desplazamiento paralelo no varía por características socioeconómicas. Además, encontramos poca evidencia para sugerir que el sesgo de respuesta inducido por el cambio en la pregunta esté caracterizado por un desplazamiento de los puntos de corte; nuestros resultados no indican que las posiciones relativas de los puntos de corte varíen de forma determinada por las características socioeconómicas individuales.

\section{Referencias}

[1] ADAMS, P., HURD, M.D, MCFADDEN, D., MERRILL, A., y RIBEIRO, T. (2003). Healthy, wealthy and wise? Tests for direct causal paths between health and socioeconomic status. Journal of Econometrics 112: 3-56.

[2] BENZEVAL, M., TAYLOR, J., y JUDGE, K. (2000). Evidence on the relationship between low income and poor health: Is the Government doing enough?. Fiscal Studies, 21(3): 375-399.

[3] BOUND, J (1991). Self-Reported versus objective measures of health in retirement models. The Journal of Human Resources, 26, 106-137.

[4] BUCK N., GERSHUNY J., ROSE D., SCOTT J. (1994). Changing Households. The BHPS 1990-1992. ESRC Research Centre on Micro-Social Change.

[5] BURSTRÖM, B. y FREDLUND, P. (2001). Self rated health: is it as good a predictor of subsequent mortality among adults in lower as well as in higher social classes?. Journal of Epidemiology and Community Health, 55: 836-840.

[6] BUTLER J.S., RICHARD V. BURKHAUSER, JENA M MITCHEL Y THEODORE P. PINCUS (1987). Measurement error in relf-reported health variables. The Review of Economics and Statistics, 69 (4): 644-650.

[7] CONTOYANNIS, P. y JONES, A. (2004). Socio-economic status, health and lifestyle. Journal of Health Economics, 23: 965-995.

[8] CONTOYANNIS P., JONES A. M., y RICE N. (2004). The dynamics of health in the British Household Panel Survey. Journal of Applied Econometrics, 19: 473-503.

[9] CROSSLEY T.F. y KENNEDY S. (2002). The reliability of self-assessed health status. Journal of Health Economics, 21: 643-658.

[10] CURRIE, J. y MADRIAN, B.C. (1999). Health, health insurance and the labor market. En Ashenfelter, O. and card, D. (eds.) Handbook of Labour Economics, Vol.3, Elsevier, Amsterdam.

[11] DEATON, A..S. y PAXSON, C.H. (1998). Ageing and inequality in income and health. American Economic Review, Papers and Proceedings 88: 248-253.

[12] ETTNER, S. (1996). New evidence on the relationship between income and health. Journal of Health Economics, 15: 67-85.

[13] FRIJTERS, P., HAISKEN-DENEW, J.P. y SHIELDS, M.A. (2003). Estimating the causal effect of income on health: evidence from post reunification East Germany, Centre for Economic Policy Discussion Paper No. 465, Australian National University. 
[14] GROOT, W. (2000). Adaptation and scale of reference bias in self-assessments of quality of life. Journal of Health Economics 19(3), 403-420.

[15] GROOTENDORST, P., FEENY, D., y FURLONG, W. (1997). Does it matter whom and how you ask? Inter and intra-rater agreement in the Ontario health survey. Journal of Clinical Epidemiology 50: 127-136.

[16] IDLER, E. L. y Y. BENYAMINI (1997). Self-rated health and mortality: a review of twenty-seven community studies. Journal of Health and Social Behavior 38(1): 21-37.

[17] IDLER, E.L. y KASL, S.V. (1995). Self-ratings of health: do they also predict change in functional ability? Journal of Gerontology 50B: S344-S353.

[18] JENKINSON, C. J. et al. (1996). The U.K. SF-36: an analysis and interpretation manual. A guide to health status measurement with particular reference to the Short Form 36 Health Survey. Oxford University, Department of Public Health and Primary Care.

[19] JONES, A.M. y LÓPEZ-NICOLÁS, A. (2004). Measurement and explanation of socioeconomic inequality in health with longitudinal data. Health Economics, 13: 10151030.

[20] KAPTEIN, A, JP SMITH, A VAN SOEST (2004). Self-reported work disability in the US and The Netherlands. RAND Labor and Population Working Paper.

[21] KENKEL, D. (1995). Should you eat breakfast? Estimates form health production functions. Health Economics, 4: 15-29.

[22] KERKHOFS, M., LINDEBOOM, M. (1995). Subjective health measures and state dependent reporting errors. Health Economics 4: 221-235.

[23] KNÄUPER BARBEL y PATRICIA A TURNER (2000). Measuring health: improving the validity of health assessments. En J. Dunbar-Jacob (Ed.), Quality of Life: In the Eye of the Beholder. University of Pittsburgh: CRCD.

[24] LINDEBOOM M (2006). Health and work of older workers. En A.M.Jones (Ed.) Elgar Companion to Health Economics: Edward Elgar, Cheltenham.

[25] LINDEBOOM M. y VAN DOORSLAER E. (2004). Cut-point shift and index shift in self-reported health. Journal of Health Economics, 23: 1083-1099.

[26] LINDLEY, J. y LORGELLY, P.K. (2003). The relative income hypothesis: does it exist over time? Evidence from the BHPS. Paper prepared for the Health Economists' Study Group. Winter 2003, Leeds.

[27] LONG, J. SCOTT (1997). Regression models for categorical and limited dependent variables. Sage Publications.

[28] MCKELVEY R.D. y ZAVOINA W. (1975). A statistical model for the analysis of ordinal level dependent variables. Journal of Mathematical Sociology, 4: 103-120.

[29] MURRAY, CJL, A TANDON, J SALOMON y CD MATHERS (2001). Enhancing cross-population comparability of survey results, GPE Discussion Paper Nr 35, WHO/EIP, Geneva.

[30] SADANA, R, CD MATHERS, AD LOPEZ, CJL MURRAY y K IBURG (2000), Comparative analysis of more than 50 household surveys on health status, GPE Discussion Paper No 15, EIP/GPE/EBD, World Health organisation, Geneva.

[31] SALAS, C. (2002). On the empirical association between poor health and low socioeconomic status at old age. Health Economics, 11: 207-220.

[32] SMITH, J.P. (1999). Healthy bodies and thick wallets: the dual relationship between health and economic status. Journal of Economic Perspectives 13: 145-166. 
[33] TERZA, J.V. (1985). Ordinal Probit: A Generalization. Communications on Statistics - Theory and Methods, 14 (1): 1-11.

[34] TAYLOR, M., BRICE, J., BUCK, N., y PRENTICE, E. (1998). British Household Panel Survey User Manual Volume A: Introduction, Technical Report and Appendices. University of Essex, Colchester.

[35] VAN DOORSLAER, E. (1987). Health, knowledge and the demand for medical care. Assen, Maastricht.

[36] VAN DOORSLAER, E. y GERDTHAM, U-G. (2003). Does inequality in self-assessed health predict inequality in survival by income? Evidence from Swedish data, Social Science and Medicine, 57: 1621-1629.

[37] VAN DOORSLAER, E. y KOOLMAN, X. (2004). Explaining the differences in income-related health inequalities across European countries. Health Economics, 13: 609-628.

[38] VAN DOORSLAER,E. y JONES, A. M. (2003). Inequalities in self-reported health: validation of a new approach to measurement. Journal of Health Economics, 22(1): 61-87.

[39] VAN DOORSLAER, E., JONES, A.M. y KOOLMAN, X. (2004). Explaining income-related inequalities in doctor utilisation in Europe. Health Economics, 13: 629647.

[40] VAN DOORSLAER, E. et al. (1997). Income-related inequalities in health: some international comparisons. Journal of Health Economics, 16 (1): 93-112.

[41] VAN DOORSLAER, E., et al. (2000). Equity in the delivery of health care in Europe and the US. Journal of Health Economics, 19: 553-583.

[42] VAN OURTI, T. (2003). Socio-economic inequality in ill-health amongst the elderly. Should one use current income or permanent income? Journal of Health Economics, 22: 187-217.

[43] VERBEEK M. (2000). Modern econometrics. Wiley.

[44] WOOLDRIDGE, J. (2002). Econometric analysis of cross section and panel data. MIT Press.

[45] ZEGER, S. L., K.-Y. LIANG y P.A. ALBERT (1988). Models for longitudinal data: a generalized estimating equation approach. Biometrics, 44: 1049-60. 
